\title{
Logarithmic spin, logarithmic rate and material frame-indifferent generalized plasticity
}

\author{
D. Soldatos \\ Department of Civil Engineering, Demokritos University of Thrace \\ 12 Vasilissis Sofias Street, Xanthi, 67100, Greece \\ S. P. Triantafyllou* \\ Department of Civil Engineering, The University of Nottingham, University Park \\ Nottingham, NG72RD, UK
}

\begin{abstract}
In this work we present a new rate type formulation of large deformation generalized plasticity which is based on the consistent use of the logarithmic rate concept. For this purpose, the basic constitutive equations are initially established in a local rotationally neutralized configuration which is defined by the logarithmic spin. These are then rephrased in their spatial form, by employing some standard concepts from the tensor analysis on manifolds. Such an approach, besides being compatible with the notion of (hyper)elasticity, offers three basic advantages, namely:
\end{abstract}

(i) The principle of material frame-indifference is trivially satisfied.

(ii) The structure of the infinitesimal theory remains essentially unaltered.

(iii) The formulation does not preclude anisotropic response.

A general integration scheme for the computational implementation of generalized plasticity models which are based on the logarithmic rate is also discussed. The performance of the scheme is tested by two representative numerical examples.

Keywords: Generalized plasticity; logarithmic rate; rotationally neutralized configuration; incrementally objective algorithm.

\section{Introduction}

Since the time of its initial introduction as "a simple theory of plasticity" Lubliner, 1974], generalized plasticity has been further elaborated in order to deal with the yield surface concept Lubliner, 1975, an axiomatic structure Lubliner. 1980], the maximum plastic dissipation postulate Lubliner, 1984, non-isothermal behavior [Lubliner, 1987], spatial covariance [Panoskaltsis et al., 2008b] and invariance principles (Panoskaltsis et al., 2011]. The theory has been also used as a

*Department of Civil Engineering, The University of Nottingham, University Park Nottingham, NG72RD, UK 
practical way of describing the elastic-plastic behavior of metallic materials in both the infinitesimal, (see, e.g., Lubliner 1991; Lubliner et al. 1993]; Auricchio and Taylor [1995]) and the finite regimes, (see, e.g., Panoskaltsis et al. 2008b a. 2011]).

The paper which constitutes the point of departure for the present work is that of Panoskaltsis et al. 2008a. In this, the authors proposed an Eulerian (spatial) formulation of the theory which was based on the consistent use of the Lie derivative concept (see, e.g., Bishop and Goldberg, 1980, pp. 128-132]; Abraham et al., 1988 , pp. 359-376]; Szekeres |2004 pp. 436-440]). The formulation was developed in a rate form by considering the additive decomposition of the rate of deformation tensor $\mathbf{d}$ into elastic $\left(\mathbf{d}_{\mathbf{e}}\right)$ and plastic $\left(\mathbf{d}_{\mathbf{p}}\right)$ parts (see, e.g., Nemat-Nasser [1982]; see also Simo and Hughes, 1998, pp. 269-271]), that is

$$
\mathbf{d}=\mathbf{d}_{\mathrm{e}}+\mathbf{d}_{\mathrm{p}}
$$

as a basic kinematic assumption. The elastic response therein has been assumed to be given by a rate constitutive equation (see, e.g., Truesdell [1955; Rivlin 1997]) as

$$
L_{\mathbf{v}} \boldsymbol{\tau}=\mathbf{a}(\boldsymbol{\tau}): \mathbf{d}_{\mathbf{e}}
$$

where $\boldsymbol{\tau}$ is the Kirchhoff stress tensor, $\mathbf{a}$ is the isotropic elastic moduli rank-4 tensor and $L_{\mathbf{v}}(\cdot)$ stands for the Lie derivative (see also Marsden and Hughes, 1994, pp. 93-104]; Stumpf and Hoppe 1997]; Simo and Hughes, 1998, pp. 254-255]). This approach offered several substantial advantages for the formulation of an elasticplastic theory in the finite deformation regime, in the sense that:

(i) Classical plasticity was included as a special case.

(ii) Some anomalies in the solution of the finite shear problem such as the "shear oscillatory phenomenon" did not appear (see, e.g., Nagtegaal and De Jong 1982, Dafalias 1983, Atluri 1984, Liu and Hong 2001).

(iii) The approach could be extended to a covariant one in a straight forward manner Simo, 1988, Panoskaltsis et al. 2008b.

Nevertheless, this formulation placed a strong restriction on the basic state functions by requiring them to be isotropic. This requirement stems from the principle of material frame-indifference (see, e.g., Noll [1973]; see also the very recent developments given in Frewer 2009, Dafalias 2011, Liu and Sampaio 2014]). Moreover, special care had to be undertaken in the selection of the rate elastic constitutive equation (2) for the approach to be compatible with the notion of hyperelasticity (see, e.g., Simo and Pister [1984) which requires the existence of a stored energy function. Elasticity without a stored energy function is difficult to motivate physically, since it may result in aberrant elastic behavior which may be manifested by 
hysteretic energy dissipation (see, e.g., Bernstein 1960) and/or residual stresses after a closed strain path (see, e.g., Lin 2002; Lin et al. 2003, Meyers et al. 2003). In this work, the large strain generalized plasticity theory is revisited and further extended by introducing the concept of the logarithmic rate that treats the aforementioned inconsistency.

Inspired by this questionable nature of the elastic response, Bruhns et al. 1999 suggested a new Eulerian rate type formulation for the description of isotropic elastic-plastic behaviour which was based on the introduction of the rather newly discovered concept of the logarithmic rate, (see, e.g., Lehmann et al. 1991; Reinhardt and Dubey 1996; Xiao et al. 1997]). More specifically, Xiao et al. 1997 proved that there exists a smooth antisymmetric tensor, the logarithmic spin $\mathbf{\Omega}^{\log }$, and an Eulerian strain measure, the Hencky logarithmic strain e, such as the time derivative of $\mathbf{e}$ in a frame of reference which spins with $\boldsymbol{\Omega}^{\log }$ is equal to the rate of deformation tensor, that is

$$
\mathbf{d}=\dot{\mathbf{e}}+\mathbf{e} \mathbf{\Omega}^{\log }-\mathbf{\Omega}^{\log } \mathbf{e}
$$

Moreover, by exploiting Eq. (3) an explicit expression for $\Omega^{\log }$ was derived Xiao et al. 1997 in terms of the spin (vorticity) tensor $\mathbf{w}$ and the principal values $b_{\mathrm{i}}$ of the left Cauchy-Green tensor $\mathbf{b}$, that is

$$
\mathbf{\Omega}^{\log }=\mathbf{w}+\mathbf{N}^{\log },
$$

where $\mathbf{N}^{\log }$ is the (spatial) antisymmetric tensor

$$
\mathbf{N}^{\log }= \begin{cases}\mathbf{O} & \text { if } b_{1}=b_{2}=b_{3} \\ \boldsymbol{\nu}[\mathbf{b}, \mathbf{d}] & \text { if } b_{1} \neq b_{2}=b_{3} \\ \nu_{1}[\mathbf{b}, \mathbf{d}]+\nu_{2}\left[\mathbf{b}^{2}, \mathbf{d}\right]+\nu_{3}\left[\mathbf{b}^{2}, \mathbf{d}, \mathbf{b}\right] & \text { if } b_{1} \neq b_{2} \neq b_{3} \neq b_{1}\end{cases}
$$

in which

$$
\begin{gathered}
\boldsymbol{\nu}=\frac{1}{b_{1}-b_{2}}\left[\frac{1+b_{1} / b_{2}}{1-b_{1} / b_{2}}+\frac{2}{\ln \left(b_{1} / b_{2}\right)}\right] \\
\nu_{\mathrm{k}}=-\frac{1}{\Delta} \sum_{\mathrm{i}=1}^{3}-\left(b_{\mathrm{i}}\right)^{3-\mathrm{k}}\left[\frac{1+\varepsilon_{\mathrm{i}}}{1-\varepsilon_{\mathrm{i}}}+\frac{2}{\ln \left(\varepsilon_{\mathrm{i}}\right)}\right], \mathrm{k}=1,2,3, \\
\varepsilon_{1}=\frac{b_{2}}{b_{3}}, \varepsilon_{2}=\frac{b_{3}}{b_{1}}, \varepsilon_{3}=\frac{b_{1}}{b_{2}}, \Delta=\left(\mathrm{b}_{1}-\mathrm{b}_{2}\right)\left(\mathrm{b}_{2}-\mathrm{b}_{3}\right)\left(\mathrm{b}_{3}-\mathrm{b}_{1}\right),
\end{gathered}
$$

Furthermore, $[\mathbf{b}, \mathbf{d}]$ in relation (5) is the Lie bracket (matrix commutator) of $\mathbf{b}$ and $\mathbf{d}$, i.e. $[\mathbf{b}, \mathbf{d}]=\mathbf{b d}-\mathbf{d} \mathbf{b}$ while the triple bracket is defined as $\left[\mathbf{b}^{2}, \mathbf{d}, \mathbf{b}\right]=$ $\mathbf{b}^{2} \mathbf{d b}-\mathbf{b d b}^{2}$. In turn, Bruhns et al. 1999. suggested that the rate constitutive equation (2) can be replaced by the rate equation 


$$
\mathbf{d}_{\mathbf{e}}=\mathbf{h}(\boldsymbol{\tau}): \hat{\boldsymbol{\tau}}^{\log },
$$

where $\hat{\boldsymbol{\tau}}^{\log }$ is the logarithmic rate of the Kirchhoff stress $\boldsymbol{\tau}$ defined as

$$
\hat{\tau}^{\log }=\dot{\tau}+\tau \Omega^{\log }-\boldsymbol{\Omega}^{\log } \tau,
$$

and $\mathbf{h}(\boldsymbol{\tau})$ is an isotropic function of $\boldsymbol{\tau}$. Accordingly, they proved that when $\mathbf{d}=\mathbf{d}_{\mathbf{e}}$, Eq. (6) is exactly integrable - i.e. there exists a scalar function $\sigma=\hat{\sigma}(\boldsymbol{\tau})$ such as $\mathbf{h}(\boldsymbol{\tau})=\frac{\partial^{2} \sigma}{\partial \boldsymbol{\tau} \partial \boldsymbol{\tau}}$. This means that upon integration, Eq. (6) results in a hyperelastic constitutive equation for the Hencky logarithmic strain tensor, that is

$$
\mathbf{e}=\frac{\partial \sigma}{\partial \boldsymbol{\tau}}
$$

These ideas led to the concept of the self-consistent (elastic-plastic) model, which may be defined as one which in the absence of plastic deformation, results in hyperelastic (conservative) response Bruhns et al. 1999. Using these derivations as a starting point further research has been conducted dealing with the elastic-plastic torsion problem Bruhns et al. 2001, the application of the Sturm's comparison theorem in the finite shear problem Liu and Hong 2001] and the Lie symmetries of the governing equations Liu, 2004. Very recent developments are those by Zhu et al. 2014; Xiao et al. 2014 dealing with the constitutive modeling of metals under cyclic loadings and Brepols et al. 2014 dealing with a material model which can be used in industrial metal forming processes. Related is also the recent work by Shutov and Ihlemann 2014], where the idea of the logarithmic rate is discussed within a recently introduced symmetry concept, namely that of weak invariance.

In this work, the concept of the logarithmic rate is introduced within the generalized plasticity framework. In addition, a general integration scheme is established for the computational implementation of generalized plasticity models.

The introduction of the logarithmic rate in the generalized plasticity is based on the defining property of the logarithmic spin and the corresponding logarithmic rotation $\mathbf{R}^{\log }$, that is by exploiting the solutions of the evolution equation

$$
\begin{aligned}
\boldsymbol{\Omega}^{\log }(\mathbf{x}, t) & =\dot{\mathbf{R}}^{\log \mathbf{T}}(\mathbf{x}, t) \mathbf{R}^{\log }(\mathbf{x}, t), \\
\mathbf{R}^{\log }(\mathbf{x}, 0) & =\mathbf{I},
\end{aligned}
$$

where $\mathbf{x}$ stand for spatial coordinates and $t$ is the time. In this way, a local rotationally neutralized configuration is introduced which is unaffected by rigid body motions superposed onto the spatial configuration. As a result any tensorial quantity defined in this configuration will be material frame-indifferent such that the homonym principle is trivially satisfied.

Next, motivated by the material rotated description of elasticity (see Green and McInnis 1967; ; Simo and Marsden 1984a b]; see also [Simo and Hughes, 1998, pp. 271-275] for the elastic-plastic case), this configuration is identified as a reference 
one for the development of the theory. The approach presented herein, besides enforcing automatically material frame-indifference, has two additional advantages, namely

(i) The structure of the infinitesimal theory remains essentially unaltered.

(ii) The formulation does not preclude anisotropic response.

The computational implementation procedure is underlined by the interpretation of the logarithmic rate as a Lie derivative; as a direct consequence the proposed scheme falls within the context of the so-called incrementally objective algorithms Hughes and Winget, 1980, Rubinstein and Atluri, 1983: Pinsky et al., 1983. The performance of the scheme is tested by two representative numerical examples.

This manuscript is organized as follows. In Section 2, the basic relations and the corresponding notions implemented in this work are introduced to facilitate subsequent derivations. Next, the concept of the logarithmic strain rate is introduced within the generalized plasticity framework and the corresponding material frameindifferent constitutive relations are presented in Section 3. The computational aspects of the proposed formulation are presented in Section 4, where a methodology is proposed for the integration of the derived governing equations. Finally, applications are presented in Section 5 to demonstrate the efficiency and versatility of the proposed formulation.

\section{Notation-Basic Relations}

As a starting point we consider a homogeneous body which occupies a region $\boldsymbol{\Omega}$ in the Euclidean ambient space $\mathbf{E}^{3}$ with points $\mathbf{X}$ labeled by $\left(X^{1}, X^{2}, X^{3}\right)$. The region $\Omega$ is identified by the body reference (material) configuration and we define a motion of the body within $\mathbf{E}^{3}$ as an one-parameter family of mappings

$$
\varphi_{\mathbf{t}}: \Omega \rightarrow \mathbf{E}^{3}, \mathbf{X}=\varphi_{t}(\mathbf{X})=\varphi(\mathbf{X}, t) \text {, i.e., } x_{i}=\varphi_{i}\left(X_{I}, t\right)=x_{i}\left(X_{I}, t\right) .
$$

Since in this work we deal with large plastic deformations and material frameindifference, it is advantageous to follow a geometrical approach (see, e.g., Marsden and Hughes, 1994 pp. 25-75,93-119]; Stumpf and Hoppe 1997) and consider both $\boldsymbol{\Omega}$ and $\mathbf{E}^{3}$ as (Riemannian) manifolds with metrics the Euclidean ones $\mathbf{I}$ and $\mathbf{i}$ respectively (see, e.g., Bishop and Goldberg, 1980, pp. 22-23]; [Szekeres, 2004, p. 413]).

The deformation gradient is the two point tensor defined as the tangent map of relation (9), that is

$$
\mathbf{F}=\mathrm{T} \varphi: \mathrm{T}_{\mathbf{X}} \boldsymbol{\Omega} \rightarrow \mathrm{T}_{\mathbf{x}} \mathbf{E}^{3},
$$

i.e.

$$
\mathbf{F}(\mathbf{X} . t)=\frac{\partial \varphi(\mathbf{X}, t)}{\partial t}, \text { i.e., } F_{i I}=\frac{\partial \varphi_{i}\left(X_{A}, t\right)}{\partial X_{I}}=\frac{\partial x_{i}\left(X_{A}, t\right)}{\partial X_{I}}
$$


where $\mathrm{T}_{\mathbf{X}} \boldsymbol{\Omega}$ and $\mathrm{T}_{\mathbf{x}} \mathbf{E}^{3}$ stand for the tangent spaces at $\mathbf{X} \in \boldsymbol{\Omega}$ and $\mathbf{x} \in \mathbf{E}^{3}$, respectively. The mapping $\varphi_{\mathbf{t}}$ is assumed invertible and orientation preserving, i.e., $J=\operatorname{det} \mathbf{F}(\mathbf{X}, t)>0$, where $J$ is the determinant of $\mathbf{F}$. The deformation gradient $\mathbf{F}$ maps the material line element $d \mathbf{X} \in \mathrm{T}_{\mathbf{X}} \boldsymbol{\Omega}$ to the ambient space (spatial) element $d \mathbf{x} \in \mathrm{T}_{\mathbf{x}} \mathbf{E}^{3}$ such as

$$
d \mathbf{x}=\mathbf{F} d \mathbf{X}, \text { i.e., } d x_{i}=\frac{\partial x_{i}}{\partial X_{I}} d X_{I} .
$$

Furthermore, the material velocity $\mathbf{V}: \Omega \rightarrow \mathbb{R}^{3}$ is defined as

$$
\mathbf{V}_{\mathrm{t}}(\mathbf{X})=\mathbf{V}(\mathbf{X}, t)=\frac{\partial \varphi(\mathbf{X}, t)}{\partial t},
$$

while the spatial velocity $\mathbf{v}: \varphi_{t}(\boldsymbol{\Omega}) \rightarrow \mathbb{R}^{3}$ is defined as

$$
\mathbf{v}_{t}=\mathbf{V}_{t} \circ \varphi_{t}^{-1} .
$$

The velocity gradient is defined as the 2-rank tensor $\mathbf{l}: \mathrm{T}_{\mathbf{x}} \mathbf{E}^{3} \times \mathrm{T}_{\mathbf{x}} \mathbf{E}^{3} \rightarrow \mathbb{R}$ as

$$
\mathbf{l}=\frac{\partial \mathbf{v}}{\partial \mathbf{x}} \text {, i.e., } \mathrm{l}_{i j}=\frac{\partial v_{i}}{\partial x_{j}} \text { or } \mathbf{l}=\dot{\mathbf{F}} \mathbf{F}^{-1} \text {, i.e, } 1_{i j}=\dot{F}_{i I} F_{I j}
$$

which can be additively decomposed into the symmetric rate of deformation tensor $\mathbf{d}: \mathrm{T}_{\mathbf{x}} \mathbf{E}^{3} \times \mathrm{T}_{\mathbf{x}} \mathbf{E}^{3} \rightarrow \mathbb{R}$ and the antisymmetric spin tensor $\mathbf{w}: \mathrm{T}_{\mathbf{x}} \mathbf{E}^{3} \times \mathrm{T}_{\mathbf{x}} \mathbf{E}^{3} \rightarrow \mathbb{R}$ such as the standard relations

$$
\mathbf{l}=\mathbf{d}+\mathbf{w}, \mathbf{d}=\frac{1}{2}\left(\mathbf{l}+\mathbf{l}^{\mathbf{T}}\right), \mathbf{w}=\frac{1}{2}\left(\mathbf{l}-\mathbf{l}^{\mathbf{T}}\right)
$$

hold.

The polar decomposition theorem states that for each $\mathbf{X} \in \boldsymbol{\Omega}$ there exists an orthogonal transformation $\mathbf{R}(\mathbf{X}): \mathrm{T}_{\mathbf{X}} \boldsymbol{\Omega} \rightarrow T_{\mathbf{X}} E$, i.e., $\mathbf{R}(\mathbf{X})^{\mathbf{T}} \mathbf{R}(\mathbf{X})=$ $\mathbf{I}, \mathbf{R}(\mathbf{X}) \mathbf{R}(\mathbf{X})^{\mathbf{T}}=\mathbf{i}$ such that

$$
\begin{aligned}
& \mathbf{F}=\mathbf{R U}, \text { i.e., } \mathbf{F}(\mathbf{X})=\mathbf{R}(\mathbf{X}) \circ \mathbf{U}(\mathbf{X}) \\
& \mathbf{F}=\mathbf{V R}, \text { i.e., } \mathbf{F}(\mathbf{X})=\mathbf{V}(\mathbf{X}) \circ \mathbf{R}(\mathbf{X})
\end{aligned}
$$

where $\mathbf{R}$ is the rotation tensor and $\mathbf{U}: \mathrm{T}_{\mathbf{X}} \boldsymbol{\Omega} \times \mathrm{T}_{\mathbf{X}} \boldsymbol{\Omega} \rightarrow \mathbb{R}, \mathbf{V}: \mathrm{T}_{\mathbf{X}} \mathbf{E}^{3} \times \mathrm{T}_{\mathbf{x}} \mathbf{E}^{3} \rightarrow \mathbb{R}$ stand for symmetric positive-definite tensors which are known as the right and left stretch tensors, respectively.

Any spatial antisymmetric tensor $\boldsymbol{\Omega}^{\prime}: \mathrm{T}_{\mathbf{x}} \mathbf{E}^{3} \times \mathrm{T}_{\mathbf{x}} \mathbf{E}^{3} \rightarrow \mathbb{R}$ constitutes an infinitesimal generator of a one parameter subgroup $G$ of the special orthogonal group $S O(3, \mathbb{R})$ (see, e.g., Szekeres, 2004, p. 170-172]), which is defined by means of the following evolution equation

$$
\begin{aligned}
& \mathbf{\Omega}^{\prime}(\mathbf{x}, t)=\dot{\mathbf{R}}^{\prime \mathbf{T}}(\mathbf{x}, t) \mathbf{R}^{\prime}(\mathbf{x}, t), \\
& \mathbf{R}^{\prime}(\mathbf{x}, 0)=\mathbf{I},
\end{aligned}
$$


In turn, the group $G$, that is

$$
G=\left\{\mathbf{R}^{\prime} \in S O(3, \mathbb{R}) / \mathbf{R}^{\prime} \text { is a solution of Eq. } 111\right\}
$$

has a (left) action on the ambient space $E$ which can be interpreted in two physically different - but mathematically equivalent - ways (see Frewer [2009], Dafalias 2011]). These are related by the so-called alias-alibi viewpoint of coordinates in differentiable manifolds, (see, e.g., Bishop and Goldberg, 1980, p. 72]). According to the alias point of view, the group action is interpreted as a (time-dependent) change of the spatial basis so that the material line element $d \mathbf{x}$ in the new (primed) basis is perceived as

$$
d \mathbf{x}^{\prime}=\mathbf{R}^{\prime} d \mathbf{x} \text {, i.e., } d x_{j^{\prime}}^{\prime}=R_{j^{\prime} i}^{\prime} d x_{i},
$$

while a n-rank tensor $\mathbf{a}: \underbrace{\mathrm{T}_{\mathbf{x}} \mathbf{E}^{3} \times \ldots \times \mathrm{T}_{\mathbf{x}} \mathbf{E}^{3}}_{\text {ncopies }} \rightarrow \mathbb{R}$ with components $\mathrm{a}_{i_{1} \ldots i_{\mathrm{n}}}$ is perceived as

$$
\mathrm{a}_{j^{\prime}{ }_{1} \ldots j^{\prime}{ }_{\mathrm{n}}}=R_{j^{\prime}{ }_{1} i_{1}}^{\prime} \ldots R_{j^{\prime}{ }_{\mathrm{n}} i_{\mathrm{n}}}^{\prime} \mathrm{a}_{i_{1} \ldots i_{\mathrm{n}}} .
$$

On the other hand, according to the alibi point of view the group action is interpreted as a remapping of the ambient space $\mathbf{E}^{3}$, that is

$$
\mathbf{x}^{\prime}=\mathbf{x}_{t}^{\prime}(\mathbf{x})=\mathbf{x}^{\prime}(\mathbf{x}, t) \text {, i.e., } x_{i}^{\prime}=x_{i}^{\prime}\left(x_{a}, t\right)
$$

with tangent map

$$
\mathbf{R}^{\prime}=\mathrm{Tx}^{\prime}: \mathrm{T}_{\mathbf{x}} \mathbf{E}^{3} \rightarrow \mathrm{T}_{\mathbf{x}^{\prime}} E .
$$

Accordingly, it may be assumed that Eqs. (13) and (14) define locally a second (spatial) configuration $\omega^{\prime}$ which is perceived from the original spatial basis. Since $\mathbf{R}^{\prime}$ is an orthogonal tensor, the inverse mapping

$$
\mathbf{x}=\mathbf{x}_{t}\left(\mathbf{x}^{\prime}\right)=\mathbf{x}\left(\mathbf{x}^{\prime}, t\right) \text {, i.e., } x_{i}=x_{i}\left(x_{b}^{\prime}, t\right),
$$

always exists and $\mathbf{R}^{\prime}$ maps the spatial line element $d \mathbf{x} \in \mathrm{T}_{\mathbf{x}} E$ to the (spatial) line element $d \mathbf{x}^{\prime} \in \mathrm{T}_{\mathbf{x}^{\prime}} \omega^{\prime}$ such as the relations

$$
d \mathbf{x}^{\prime}=\mathbf{R}^{\prime} d \mathbf{x} \text {, i.e., } d x_{i}^{\prime}=R_{i j}^{\prime} d x_{j},
$$

and

$$
d \mathbf{x}=\mathbf{R}^{\prime \mathbf{T}} d \mathbf{x}^{\prime} \text {, i.e., } d x_{i}=R_{i j}^{\prime} d x_{j}^{\prime},
$$

exist for all $t$ in the domain $0 \leq t \leq t_{c}$ for all finite $t_{c}$. 
The push-forward $\mathbf{x}^{\prime}{ }_{*}(\mathbf{a})$ of the tensor a (see, e.g., Abraham et al., 1988, pp. 265,266]; Marsden and Hughes, 1994, p.67]; Stumpf and Hoppe 1997) is the (nrank) tensor $\mathbf{a}^{\prime}: \underbrace{T_{\mathbf{x}^{\prime}} \omega^{\prime} \times \ldots \times T_{\mathbf{x}^{\prime}} \omega^{\prime}}_{\text {ncopies }} \rightarrow \mathbb{R}$ defined in the primed configuration as

$$
\mathrm{a}_{i_{1} \ldots i_{\mathrm{n}}}^{\prime}=R_{i_{1} j_{1}}^{\prime} \ldots R_{i_{\mathrm{n}} j_{\mathrm{n}}}^{\prime} \mathrm{a}_{j_{1} \ldots j_{\mathrm{n}}}
$$

while the pull-back of tensor $\mathbf{a}^{\prime}$ is defined to be the spatial tensor

$$
\mathbf{x}^{\prime *}(\mathbf{a})=\mathbf{x}_{*}^{\prime-1}(\mathbf{a})=\mathbf{x}_{*}(\mathbf{a}) .
$$

The salient feature of this paper relies crucially on the fact that the primed configuration which is defined by the orthogonal group 12 , is a rotationally relaxed (neutralized) one and accordingly it will be unaffected by rigid body motions superposed onto the (original) spatial configuration. As a direct consequence the principle of material frame-indifference will be trivially satisfied in this configuration, so that the later can play the role of a reference configuration for the development of an elastic-plastic theory. This will become clear in the forthcoming section where the theory of rate-independent generalized plasticity is developed in a rotationally neutralized configuration which is defined by means of the logarithmic spin.

\section{Material frame-indifferent generalized plasticity}

Since the theory presented in this work is based on the concept of the logarithmic spin, we identify the spin tensor $\Omega^{\prime}$ by $\Omega^{\log }$, such as the corresponding reference configuration is determined by the solutions of the evolution equation (8). Then the basic kinematical assumption (Eq. (1)) in the $\mathbf{R}^{\log }$ - rotated (reference) configuration reads

$$
\mathbf{d}^{\prime}=\mathbf{d}_{\mathbf{e}}^{\prime}+\mathbf{d}_{\mathbf{p}}^{\prime}
$$

where $\mathbf{d}^{\prime}, \mathbf{d}^{\prime} \mathbf{e}$ and $\mathbf{d}_{\mathbf{p}}^{\prime}$ are the pull-backs by $\mathbf{x}$ (see Eqs. 15, 16) of $\mathbf{d}, \mathbf{d}_{\mathbf{e}}$ and $\mathbf{d}_{\mathbf{p}}$ respectively. The pull-back of $\mathbf{d}$ assumes the following form

$$
\mathbf{d}^{\prime}=\mathbf{x}^{*}(\mathbf{d})=\mathbf{R}^{\log \mathbf{T} *}(\mathbf{d})=\left(\mathbf{R}^{\log \mathbf{T}}\right)^{\mathbf{T}} \mathbf{d}\left(\mathbf{R}^{\log \mathbf{T}}\right)=\mathbf{R}^{\log } \mathbf{d} \mathbf{R}^{\log \mathbf{T}}
$$

while similar expressions hold for $\mathbf{d}^{\prime} \mathbf{e}$ and $\mathbf{d}_{\mathbf{p}}^{\prime}$, as well.

Remark 3.1. Note that the evolution Eq. (8) which determines the $\mathbf{R}^{\log }-$ rotated configuration can be written in the form

$$
\begin{aligned}
& \dot{\mathbf{R}}^{\log }(\mathbf{x}, t)=-\mathbf{R}^{\log }(\mathbf{x}, t) \mathbf{\Omega}^{\log }(\mathbf{x}, t) \\
& \mathbf{R}^{\log }(\mathbf{x}, 0)=\mathbf{I}
\end{aligned}
$$


The unique solution of the differential Eq. $18 p$ is the exponential map denoted $\exp : \mathrm{g}^{\log } \rightarrow G^{\log }$, where $\mathrm{g}^{\log }$ stands for the Lie algebra of the one-parameter subgroup

$$
G^{\log }=\left\{\mathbf{R}^{\log } \in S O(3, \mathbb{R}) / \mathbf{R}^{\log } \text { is a solution of Eg. 18) }\right\}
$$

having the ordinary matrix commutator as its Lie bracket (see, e.g., Szekeres, 2004, pp. 166-177]). The corresponding expression for $\mathbf{R}^{\log }$ reads

$$
\mathbf{R}^{\log }=\exp \left[t\left(-\boldsymbol{\Omega}^{\log }\right)\right]=\mathbf{I}-t \boldsymbol{\Omega}^{\log }+\frac{1}{2 !} t^{2}\left(\boldsymbol{\Omega}^{\log }\right)^{2}-\frac{1}{3 !} t^{3}\left(\boldsymbol{\Omega}^{\log }\right)^{3}+\ldots, \boldsymbol{\Omega}^{\log } \in \mathrm{g}^{\log } .
$$

Remark 3.2. The derivations presented in this context are based on the introduction of the new $\left(\mathbf{R}^{\log }\right.$ - rotated) configuration. Accordingly, the alibi point of view of coordinates in $\mathbf{E}^{3}$ is adopted; this approach to the logarithmic rate concept is different from the original one as it is given for instance in Xiao et al. 1997. In this, rather than considering the idea of a new configuration, the alias point of view has been adopted by identifying the original spatial coordinate system to be a "fixed background frame" and the primed system as one which spins with respect to this frame by $\Omega^{\log }$.

Generalized plasticity is a local internal variable theory of rate-independent behavior which is based on the assumption that plastic deformation takes place on loading but not on unloading Lubliner, 1974, 1975, 1980. The local state at the point $\mathbf{x}^{\prime}$ of the reference configuration is assumed to be determined by the couple $\left(\boldsymbol{\tau}^{\prime}, \mathbf{a}^{\prime}\right)$ where $\boldsymbol{\tau}^{\prime}: \mathrm{T}_{\mathbf{x}^{\prime}} \omega^{\prime} \times \mathrm{T}_{\mathbf{x}^{\prime}} \omega^{\prime} \rightarrow \mathbb{R}$ is the ( $\mathbf{R}^{\log }$-rotated) Kirchhoff stress tensor which is defined as the pull-back of the Kirchhoff stress $\boldsymbol{\tau}: \mathrm{T}_{\mathbf{x}} \mathbf{E}^{3} \times \mathrm{T}_{\mathbf{x}} \mathbf{E}^{3} \rightarrow \mathbb{R}$ i.e.

$$
\boldsymbol{\tau}^{\prime}=\mathbf{x}^{*}(\boldsymbol{\tau})=\mathbf{R}^{\log \mathbf{T} *}(\boldsymbol{\tau})=\left(\mathbf{R}^{\log \mathbf{T}}\right)^{-1} \boldsymbol{\tau}\left(\mathbf{R}^{\log }\right)^{-\mathbf{T}}=\mathbf{R}^{\log } \boldsymbol{\tau} \mathbf{R}^{\log \mathbf{T}},
$$

and $\mathbf{a}^{\prime}: \underbrace{\mathrm{T}_{\mathbf{x}^{\prime}} \omega^{\prime} \times \ldots \times \mathrm{T}_{\mathbf{x}^{\prime}} \omega^{\prime}}_{\text {ncopies }} \rightarrow 1 \mathbb{R}$ stands for the components of the internal variable vector.

A local process $\psi^{\prime}$ (at the point $\mathbf{x}^{\prime}$ ) is defined as a curve in the state space $S^{\prime} \subset\left(\mathrm{T}_{\mathbf{x}^{\prime}} \omega^{\prime} \times \mathrm{T}_{\mathbf{x}^{\prime}} \omega^{\prime}\right) \times \underbrace{\left(\mathrm{T}_{\mathbf{x}^{\prime}} \omega^{\prime} \times \ldots \times \mathrm{T}_{\mathbf{x}^{\prime}} \omega^{\prime}\right.}_{\text {ncopies }})$, i.e. as a mapping $\psi^{\prime}: I \in \mathbb{R} \rightarrow S^{\prime}$, with $\psi^{\prime}(t)=\left(\boldsymbol{\tau}^{\prime}(t), \mathbf{a}^{\prime}(t)\right)$. The direction and the speed of the process are determined by the tangent vector $\dot{\psi}^{\prime}: S^{\prime} \rightarrow \mathrm{T} S^{\prime}$, with $\dot{\psi}^{\prime}=\left(\dot{\boldsymbol{\tau}}^{\prime}, \dot{\mathbf{a}}^{\prime}\right)$, where $\mathrm{T} S^{\prime}$ is the tangent space of $S^{\prime}$. Since the stress rate $\dot{\boldsymbol{\tau}}^{\prime}$ is assumed to be known under stress control, the component $\dot{\mathbf{a}}^{\prime}$ of $\dot{\psi}^{\prime}$ has to be determined.

The determination procedure is closely tied to the concept of the elastic range (see, e.g., Lubliner [1980, 1991]), which is defined at any material state as the region in the stress space $T^{\prime} \subset S^{\prime}$ comprising the stresses which can be attained elastically (i.e. with no change in the internal variable vector) from the current stress state. It is assumed that the elastic range is a regular set in the sense that is the closure 
of an open set. The boundary of this set may be defined as a loading surface (see, e.g, Lubliner 1975, 1980, 1991]). In turn a material state may be defined as elastic if it is an interior point of its elastic range and plastic if it is a boundary point of its elastic range; in the latter case the material state lies in a loading surface. Note that the notion of a process is introduced implicitly here.

On the basis of some basic axioms and results from set theory and the involvement of Caratheodory's theorem Lubliner, 1975] - see also Lubliner, 1980, 1991 showed that the component $\dot{\mathbf{a}}^{\prime}$ of the tangent vector can be given by an equation of the form

$$
\dot{\mathbf{a}}^{\prime}=h^{\prime} \boldsymbol{\mu}^{\prime}\left(\boldsymbol{\tau}^{\prime}, \mathbf{a}^{\prime}\right)\left\langle\boldsymbol{\nu}^{\prime}: \dot{\boldsymbol{\tau}}^{\prime}\right\rangle
$$

where $h^{\prime}: S^{\prime} \rightarrow \mathbb{R}$ is a scalar function of the state variables, $\boldsymbol{\mu}^{\prime}: S^{\prime} \rightarrow \mathrm{T} S^{\prime}$ is a non-vanishing (tensorial) function, $\boldsymbol{\nu}^{\prime}$ is the outward normal to the loading surface and $\langle\cdot\rangle$ stands for the Macauley bracket defined as $\langle x\rangle=\frac{x+|x|}{2}$.

The inner product $\boldsymbol{\nu}^{\prime}: \dot{\boldsymbol{\tau}}^{\prime}$ of the tangent vector $\dot{\boldsymbol{\tau}}^{\prime} \in \mathrm{T} T^{\prime}$ with the normal vector $\boldsymbol{\nu}^{\prime}\left(\in \mathrm{T} T^{\prime}\right)$ in Eq. 21) is defined as the loading rate. The loading rate determines the velocity and the direction of a process from a plastic state relative to its elastic range. If $\boldsymbol{\nu}^{\prime}: \dot{\boldsymbol{\tau}}^{\prime}<0$, then the elastic range remains invariant under the flow of $\dot{\boldsymbol{\tau}}^{\prime}$ (see, e.g., Abraham et al., 1988, p. 257]) and the process results in elastic response. If $\boldsymbol{\nu}^{\prime}: \dot{\boldsymbol{\tau}}^{\prime}>0$, then the elastic domain is not invariant anymore, and a new plastic state at a new value of $\mathbf{a}^{\prime}$ is initiated. The limiting case, where $\boldsymbol{\nu}^{\prime}: \dot{\boldsymbol{\tau}}^{\prime}=0$, follows from the continuity of the material Lubliner, 1980 and results in elastic response; such a process is referred as neutral loading.

Furthermore, the values of the function $h^{\prime}$ in equation 21 enforce the defining property of a plastic state; accordingly these values must be positive at a plastic state and zero at an elastic one. Moreover, the set defined by the equation $h^{\prime}\left(\boldsymbol{\tau}^{\prime}, \mathbf{a}^{\prime}\right)=0$, which in turn comprises all elastic states may be defined as the elastic domain, and its boundary constitutes the yield hypersurface Lubliner, 1975 . Panoskaltsis et al., 2008b. The projection of the elastic domain onto a hyper-plane defined by $\mathbf{a}^{\prime}=$ const. is called the elastic domain at $\mathbf{a}^{\prime}$, and its boundary constitutes the yield surface. Since the elastic domain (at $\mathbf{a}^{\prime}$ ) comprises only elastic states, it is a subset of the elastic range; the particular case when these two sets coincide corresponds to classical plasticity and the yield surface coincides with the initial loading surface Lubliner, 1975; Panoskaltsis et al., 2008b.

It remains to specify an additional equation for the plastic rate of deformation tensor $\mathbf{d}_{\mathbf{p}}^{\prime}$ (flow rule). This is performed on the basis of the aforementioned analysis so that such an equation may be written as

$$
\mathbf{d}_{\mathbf{p}}^{\prime}=h^{\prime} \boldsymbol{\lambda}^{\prime}\left(\boldsymbol{\tau}^{\prime}, \mathbf{a}^{\prime}\right)\left\langle\boldsymbol{\nu}^{\prime}: \dot{\boldsymbol{\tau}}^{\prime}\right\rangle
$$

where $\boldsymbol{\lambda}^{\prime}: S \rightarrow$ T $S$ is a tensorial function of the state variables which is associated which the direction of the plastic flow. The particular case where $\boldsymbol{\lambda}^{\prime}=\boldsymbol{\nu}^{\prime}$ corresponds to normality or associative plasticity. 
The loading-unloading criteria in the rotated configuration can be defined explicitly by means of Eqs. 21) and 22 as

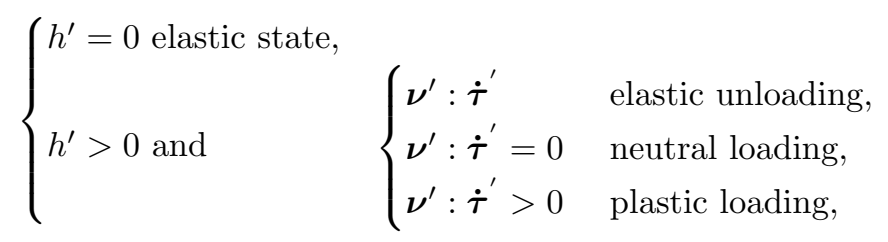

We note that the present description of generalized plasticity in the $\mathbf{R}^{\log }$-rotated configuration leaves the infinitesimal structure of the theory Lubliner. 1991], essentially unaltered. The basic difference between the two approaches has its origins in the basic kinematic assumption (17) which results in the flow rule 22 in terms of the plastic rate of deformation tensor $\mathbf{d}_{\mathbf{p}}^{\prime}$; nevertheless, this flow rule is identical to that of the infinitesimal theory - see further Lubliner 1991 - if $\mathbf{d}_{\mathbf{p}}^{\prime}$ is replaced by the rate of the infinitesimal plastic strain rate tensor $\dot{\varepsilon_{p}}$. To demonstrate the concepts discussed in this section, the following application is presented.

Example 3.1. We revisit within the present context the model discussed in Lubliner et al. 1993, which is motivated by classical metal plasticity. In this case the loading surfaces are assumed to be given by a von-Mises type expression

$$
f\left(\boldsymbol{\tau}^{\prime}, a^{\prime}, \mathbf{q}^{\prime}\right)=\left\|\boldsymbol{\tau}^{\prime}-\mathbf{q}^{\prime}\right\|-\sqrt{\frac{2}{3}}\left(\sigma_{y}^{\prime}+K a^{\prime}\right)=\text { const. },
$$

where $a^{\prime}$ is a scalar internal variable which controls the isotropic hardening of the von-Mises loading surface and $\mathbf{q}^{\prime}$ is a (purely deviatoric) tensorial internal variable, usually named back stress which defines the center of the loading surface in stress space and accordingly controls its kinematic hardening. Finally, in Eq. 23) $\sigma_{y}^{\prime}$ stands for the uniaxial ( $\mathbf{R}^{\log }$-rotated) yield stress and $K$ is the isotropic hardening modulus. The scalar function $h^{\prime}$ is assumed to be given as in Lubliner et al. 1993.

$$
h^{\prime}=\frac{\left\langle f^{\prime}\right\rangle}{(K+H) \beta+R\left(\beta-f^{\prime}\right)},
$$

where $H$ is the kinematic hardening modulus and $R$ and $\beta$ are two additional model parameters. The (associative) flow rule can be assumed to be given as

$$
\mathbf{d}_{\mathbf{p}}^{\prime}=h^{\prime} \boldsymbol{\nu}^{\prime}\left\langle\boldsymbol{\nu}^{\prime}: \dot{\boldsymbol{\tau}}^{\prime}\right\rangle
$$

while the rate equations for the internal variables are given as

$$
\begin{gathered}
\dot{a}^{\prime}=\sqrt{\frac{2}{3}} h^{\prime}\left\langle\boldsymbol{\nu}^{\prime}: \dot{\boldsymbol{\tau}}^{\prime}\right\rangle, \\
\dot{\mathbf{q}}^{\prime}=\frac{2}{3} H \mathbf{d}_{\mathbf{p}}^{\prime} .
\end{gathered}
$$


Remark 3.3. The formulation in the $\mathbf{R}^{\log }$ - rotated (reference) configuration does not preclude anisotropic response, since there is no requirement for the basic state functions $h^{\prime}, \boldsymbol{\lambda}^{\prime}$ and $\boldsymbol{\mu}^{\prime}$ to be isotropic ones, (see, e.g., [Simo and Hughes, 1998, pp. 260-261,270]). For instance, in the material model discussed in Example 3.1 the (isotropic) von-Mises expression (23) for the loading surfaces can be replaced by any other anisotropic expression. A possible choice can be a Hill's type expression which within the present context reads

$f^{\prime}\left(\boldsymbol{\tau}^{\prime}, a^{\prime}, \mathbf{q}^{\prime}\right)=\left\|\frac{1}{2} A_{i^{\prime} j^{\prime} k^{\prime} l^{\prime}}\left({\boldsymbol{\boldsymbol { \tau } ^ { \prime }}}_{{ }^{\prime} j^{\prime} j^{\prime}}-q_{{ }^{\prime} j^{\prime}}^{\prime}\right)\left({\boldsymbol{\boldsymbol { \tau } ^ { \prime }}}_{{ }^{\prime} l^{\prime}{ }^{\prime}}-q_{k^{\prime} l^{\prime}}^{\prime}\right)\right\|-\sqrt{\frac{2}{3}}\left(\sigma_{y}^{\prime}+K a^{\prime}\right)=$ const., where $\mathbf{A}$ is a 4-rank tensor which possesses the same symmetries with the elasticity tensors, (see, e.g., Simo and Hughes, 1998, pp. 256,257]).

Next, the derived theory is re-formulated in the spatial configuration, by interpreting the logarithmic rate as a Lie derivative. The procedure is illustrated through a representative example for brevity.

Example 3.2. Starting from Eq. (20), the pull- back of the Kirchhoff stress tensor $\boldsymbol{\tau}$, that is the $\mathbf{R}^{\log }$-rotated stress tensor is defined as

$$
\boldsymbol{\tau}^{\prime}=\mathbf{R}^{\log } \boldsymbol{\tau} \mathbf{R}^{\log \mathbf{T}} .
$$

while its corresponding time-derivative is derived as

$$
\dot{\boldsymbol{\tau}}^{\prime}=\dot{\mathbf{R}}^{\log } \boldsymbol{\tau} \mathbf{R}^{\log \mathbf{T}}+\mathbf{R}^{\log } \dot{\boldsymbol{\tau}} \mathbf{R}^{\log \mathbf{T}}+\mathbf{R}^{\log } \boldsymbol{\tau} \dot{\mathbf{R}}^{\log \mathbf{T}}
$$

Pushing forward $\dot{\boldsymbol{\tau}}^{\prime}$ onto the spatial configuration the following relation is established

$$
\begin{aligned}
\mathbf{x}_{*}\left(\dot{\boldsymbol{\tau}}^{\prime}\right) & =\left(\mathbf{x}^{\prime-1}\left(\dot{\boldsymbol{\tau}}^{\prime}\right)\right)=\mathbf{R}^{\log \mathbf{T}} \dot{\boldsymbol{\tau}}^{\prime} \mathbf{R}^{\log } \\
& =\mathbf{R}^{\log \mathbf{T}}\left(\dot{\mathbf{R}}^{\log } \boldsymbol{\tau} \mathbf{R}^{\log \mathbf{T}}+\mathbf{R}^{\log } \dot{\boldsymbol{\tau}} \mathbf{R}^{\log \mathbf{T}}+\mathbf{R}^{\log } \boldsymbol{\tau} \dot{\mathbf{R}}^{\log \mathbf{T}}\right) \mathbf{R}^{\log } \\
& =\dot{\boldsymbol{\tau}}+\mathbf{R}^{\log \mathbf{T}} \dot{\mathbf{R}}^{\log } \boldsymbol{\tau}+\boldsymbol{\tau} \dot{\mathbf{R}}^{\log \mathbf{T}} \mathbf{R}^{\log }
\end{aligned}
$$

which in view of Eq. (7) results in

$$
\mathbf{x}_{*}\left(\boldsymbol{\tau}^{\prime}\right)=\dot{\tau}-\boldsymbol{\Omega}^{\log } \boldsymbol{\tau}+\boldsymbol{\tau} \boldsymbol{\Omega}^{\log }
$$

that is

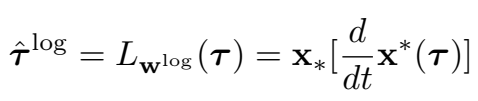

where $\mathbf{w}^{\log }: \mathbf{x}\left(\omega^{\prime}\right) \rightarrow 1 \mathbb{R}^{3}$ is the (spatial) velocity of $\mathbf{x}$ with respect to the

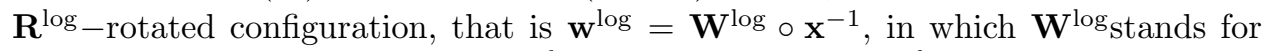
the (material) velocity in $\omega^{\prime}$ i.e. $\mathbf{W}_{\mathrm{t}}^{\log }: \omega^{\prime} \rightarrow 1 \mathbb{R}^{3}$ where $\mathbf{W}_{\mathrm{t}}^{\log }\left(\mathbf{x}^{\prime}\right)=\mathbf{W}^{\log }\left(\mathbf{x}^{\prime}, t\right)=$ $\frac{\partial \mathbf{x}\left(\mathbf{x}^{\prime}, t\right)}{\partial t}$. 
Remark 3.4. A clearer interpretation of the logarithmic rate as a Lie derivative can be given by noting that the space of 2-rank tensors $\mathrm{T}_{\mathbf{x}} \mathbf{E}^{3} \times \mathrm{T}_{\mathbf{x}} \mathbf{E}^{3} \rightarrow \mathbb{R}$, is isomorphic to the general linear group $G L(3, \mathbb{R})$ and accordingly both $\boldsymbol{\tau}$ and $\boldsymbol{\Omega}^{\log }$ can be considered as (time-dependent) vector fields in $G L(3, \mathbb{R})$. Then the logarithmic rate in terms of the Lie bracket of $\boldsymbol{\tau}$ and $\boldsymbol{\Omega}^{\log }$ reads

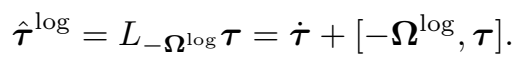

In this expression, $\dot{\tau}$ stands for the local rate of change of $\boldsymbol{\tau}$, while the (autonomous) part $\left[-\Omega^{\log }, \boldsymbol{\tau}\right]$ stands for the co-rotational rate of change of $\boldsymbol{\tau}$. Note that in the case where the alibi point of view is adopted in the description, $-\boldsymbol{\Omega}^{\log }=\boldsymbol{\Omega}^{\log \mathbf{T}}$ equals the spin of the original spatial configuration with respect to the $\mathbf{R}^{\log }-$ rotated configuration, while in the case where the alias point of view is adopted $\boldsymbol{\Omega}^{\log \mathbf{T}}$ is just the spin of the original spatial basis as perceived in the new $\left(\mathbf{R}^{\log }\right.$-rotated) one.

On the basis of these ideas we can extend the concept of the logarithmic rate in order to deal with an arbitrary $n$-rank tensor $\mathbf{a}: \underbrace{\mathrm{T}_{\mathbf{x}} \mathbf{E}^{3} \times \ldots \times \mathrm{T}_{\mathbf{x}} \mathbf{E}^{3}}_{\text {ncopies }} \rightarrow \mathbb{R}$ as

$$
\hat{\mathbf{a}}^{\log }=L_{\mathbf{w}^{\log }}(\mathbf{a})=\mathbf{x}_{*}\left[\frac{d}{d t} \mathbf{x}^{*}(\mathbf{a})\right]
$$

Note that if this is the case the logarithmic rate $\hat{\mathbf{a}}^{\log }$ is defined solely in terms of the corresponding logarithmic rotation $\mathbf{R}^{\log }$.

By means of this interpretation we can show in a straight forward manner that the primed configuration is unaffected by arbitrary rigid body motions superposed onto the spatial configuration. Such a motion, (see, e.g., Simo and Hughes, 1998, pp. 252-255]) will be of the form $\mathbf{x}^{*}=\boldsymbol{\Lambda}(t) \mathbf{x}+\mathbf{j}(t)$, where $\boldsymbol{\Lambda}(t)$ is an orthogonal tensor and $\mathbf{j}(t)$ is a vector in $\mathbf{E}^{3}$. Then the primed configuration $\omega^{\prime}$ is mapped to the stared configuration $\omega^{*}$ by means of the (orthogonal) tensor

$$
\mathbf{R}^{\log \mathbf{T}^{*}}: \mathrm{T}_{\mathbf{x}^{\prime}} \omega^{\prime} \rightarrow \mathrm{T}_{\mathbf{x}^{*} \omega^{*}}, \mathbf{R}^{\log \mathbf{T}^{*}}=\mathbf{\Lambda} \mathbf{R}^{\log \mathbf{T}}, \text {, i.e., } \mathrm{R}_{\alpha \mathrm{a}}^{\log *}=\Lambda_{\alpha i} \mathrm{R}_{i \mathrm{a}}^{\log },
$$

so that the time derivative of the n-rank tensor $\mathbf{a}^{\prime}: \underbrace{\mathrm{T}_{\mathbf{x}^{\prime}} \omega^{\prime} \times \ldots \times \mathrm{T}_{\mathbf{x}^{\prime}} \omega^{\prime}}_{\text {ncopies }} \rightarrow R$ upon the superposed motion in view of Eq. 29, , reads

$$
\begin{aligned}
\left(a^{\prime *}\right)_{a b \ldots m} & =R_{\alpha a}^{\log *} R_{\beta b}^{\log *} \ldots R_{\mu m}^{\log *}\left(\hat{a}^{\log }\right)_{\alpha \beta \ldots \mu}^{*} \\
& =\Lambda_{\alpha i} R_{i a}^{\log } \Lambda_{\beta j} R_{j b}^{\log } \ldots \Lambda_{\mu m} R_{m m}^{\log } \Lambda_{\alpha k} \Lambda_{\beta l} \ldots \Lambda_{\mu q}\left(\hat{a}^{l o g}\right)_{k l \ldots q} \\
& =\delta_{i k} \delta_{j l} \ldots \delta_{m q} R_{i a}^{\log } R_{j b}^{\log } \ldots R_{m m}^{\log \left(\hat{a}^{l o g}\right)_{k l \ldots q}} \\
& =R_{k a}^{\log } R_{l b}^{\log } \ldots R_{q m}^{\log }\left(\hat{a}^{\log }\right)_{k l \ldots q} \\
& =(\dot{a})_{a b \ldots m}
\end{aligned}
$$


Remark 3.5. $\mathbf{w}^{\log }$ can be determined by noting that $\mathbf{w}^{\log }(\mathbf{x}, t)$ is a time dependent Killing vector field (see, e.g., [Szekeres, 2004, pp. 578-583]; Marsden and Hughes, 1994, p. 19]), that is one which preserves the Euclidean metric i. The corresponding

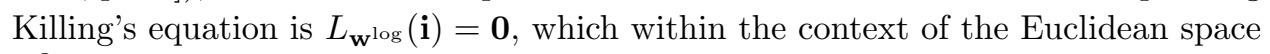
$\mathbf{E}^{3}$ can be rephrased in the form, (see, e.g., Szekeres, 2004, pp. 578, 580])

$$
\frac{\partial w_{i}^{\log }}{\partial x_{j}}+\frac{\partial w_{j}^{\log }}{\partial x_{i}}=0
$$

The solutions of this equation within the present setting are given as

$$
w_{i}^{\log }=-\Omega_{i j}^{\log } x_{j}+c_{i},
$$

where $c_{i}$ is a (time-dependent) vector field in $E$.

The general form of the mapping $\mathbf{x}=\mathbf{x}\left(\mathbf{x}^{\prime}, t\right)$ - see, e.g., problem 6.1 in p. 99 in Marsden and Hughes 1994 - can be found to be

$$
x_{i}(\mathbf{x}, t)=R_{i j}^{\log \mathbf{T}}(t) x_{j}^{\prime}(t)+d_{i}(t),
$$

where the $d_{i}{ }^{\prime} s$ are the components of a curve $d: \mathbb{R} \rightarrow E$. Note that the $\mathbf{R}^{\log }$-rotated configuration is undetermined up to a rigid body translation.

The equivalent development of the theory in the spatial configuration can be derived by performing a push-forward operation to the basic Eqs. 211 and 222 as

$$
\begin{gathered}
\hat{\mathbf{a}}^{\log }=h \boldsymbol{\mu}\left(\boldsymbol{\tau}, \mathbf{a}, \mathbf{R}^{\log }\right)\left\langle\boldsymbol{\nu}: \hat{\boldsymbol{\tau}}^{\log }\right\rangle, \\
\mathbf{d}_{\mathbf{p}}=h \boldsymbol{\lambda}\left(\boldsymbol{\tau}, \mathbf{a}, \mathbf{R}^{\log }\right)\left\langle\boldsymbol{\nu}: \hat{\boldsymbol{\tau}}^{\log }\right\rangle,
\end{gathered}
$$

where $\mathbf{a}$ and $\boldsymbol{\nu}$ stand for the push-forwards in the spatial configuration of $\mathbf{a}^{\prime}$ and $\boldsymbol{\nu}^{\prime}, h$ stands for the equivalent expression of the (scalar invariant) function $h^{\prime}$ in terms of the spatial variables $\boldsymbol{\tau}$, a and the logarithmic rotation $\mathbf{R}^{\log }$, and $\boldsymbol{\lambda}, \boldsymbol{\mu}$ are defined as the push-forwards of the functions $\boldsymbol{\lambda}^{\prime}$ and $\boldsymbol{\mu}^{\prime}$. It is noted that the (scalar invariant) loading rate $\boldsymbol{\nu}^{\prime}: \dot{\boldsymbol{\tau}}^{\prime}$ is transformed in the spatial configuration to $\boldsymbol{\nu}: \hat{\boldsymbol{\tau}}^{\log }$. We further note the presence of the logarithmic rotation tensor $\mathbf{R}^{\log }$ among the arguments of $\boldsymbol{\lambda}$ and $\boldsymbol{\mu}$ due to the push-forward operation by which Eqs. (30) and (31) have been derived from Eqs. 21) and (22), respectively. In the particular case where the functions and $\boldsymbol{\mu}^{\prime}$ are chosen to be isotropic functions of their arguments, then the expressions of $\boldsymbol{\lambda}$ and $\boldsymbol{\mu}$ will be identical to those of $\boldsymbol{\lambda}^{\prime}$ and $\boldsymbol{\mu}^{\prime}$, respectively. Finally, the loading-unloading criteria in the spatial description in view of Eqs. (30) and (31) read

$$
\begin{cases}h=0 & \text { elastic state, } \\ h>0 & \begin{cases}\boldsymbol{\nu}: \hat{\boldsymbol{\tau}}^{\log } & \text { elastic unloading } \\ \boldsymbol{\nu}: \hat{\boldsymbol{\tau}}^{\log }=0 & \text { neutral loading } \\ \boldsymbol{\nu}: \hat{\boldsymbol{\tau}}^{\log }>0 & \text { plastic loading }\end{cases} \end{cases}
$$


which are identical with those which have been discussed in the approach of Bruhns et al. 1999.

Example 3.3. The equivalent spatial setting of the model discussed in Example 3.1. can be found by a push-forward operation to Eqs. 23), 24, 25) and 26. The resulting equations are:

(i) Von-Mises type expression for the loading surfaces

$$
f(\boldsymbol{\tau}, a, \mathbf{q})=\|\boldsymbol{\tau}-\mathbf{q}\|-\sqrt{\frac{2}{3}}\left(\sigma_{y}+K a\right)=\text { const. }
$$

(ii) Associative flow rule

$$
\mathbf{d}_{\mathbf{p}}=h \boldsymbol{\nu}\left\langle\boldsymbol{\nu}: \hat{\boldsymbol{\tau}}^{\log }\right\rangle
$$

(iii) Hardening laws

$$
\begin{gathered}
\dot{a}=\sqrt{\frac{2}{3}} h\left\langle\boldsymbol{\nu}: \hat{\boldsymbol{\tau}}^{\log }\right\rangle, \\
\hat{\mathbf{q}}^{\log }=\frac{2}{3} H \mathbf{d}_{\mathbf{p}} .
\end{gathered}
$$

Since the expression for the loading surfaces is an isotropic function of the $\mathbf{R}^{\log }$-rotated variables $\boldsymbol{\tau}^{\prime}, a^{\prime}, \mathbf{q}^{\prime}$, that is

$$
f^{\prime}\left(\boldsymbol{\tau}^{\prime}, a^{\prime}, \mathbf{q}^{\prime}\right)=f^{\prime}(\boldsymbol{\tau}, a, \mathbf{q})=f(\boldsymbol{\tau}, a, \mathbf{q}),
$$

the following identities hold

$$
h=h^{\prime}, \boldsymbol{\nu}=\frac{\partial f}{\partial \boldsymbol{\tau}}=\frac{\partial f^{\prime}}{\partial \boldsymbol{\tau}^{\prime}}=\boldsymbol{\nu}^{\prime}
$$

Example 3.4. A particular case of interest arises where the function $\mathrm{h}$ is a nonvanishing (e.g. exponential, hyperbolic) function of its arguments; accordingly there are no elastic states and the elastic domain is degenerated to a single surface which may be defined as a quasi-yield surface Lubliner 1975. Such a case appears in the recent paper by Xiao et al. 2014] where the flow rule is formulated as

$$
\mathbf{d}_{\mathbf{p}}=\frac{y(\tau, a, \mathbf{q})}{\mathrm{h}(\tau, a, \mathbf{q})} \nu\left\langle\nu: \hat{\tau}^{\log }\right\rangle
$$

where $\mathrm{h}$ is a positive function of the denoted arguments - see Eq. (18) and Appendix in Xiao et al. 2014 - and the (positive) function is defined in a way that tends to zero at very small stress levels, that is

$$
y(\tau, a, \mathbf{q})=\frac{g(\tau, a, \mathbf{q})}{r(a, \mathbf{q})} \exp \left\{-m\left[1-\frac{g(\tau, a, \mathbf{q})}{r(a, \mathbf{q})}\right]\right\}
$$


where $m$ is a model parameter and $r, g$ are two additional state functions which are related to the von-Mises loading surface by a relation of the form

$$
f(\tau, a, \mathbf{q})=g(\tau, a, \mathbf{q})-r(a, \mathbf{q})
$$

Due to this formulation the model by Xiao et al. 2014 has the ability to predict plastic strains at any stress level no matter how small it is.

In closing we state the following remarks:

Remark 3.6. By recalling the basic kinematic assumption (1), the hyperelastic rate constitutive equation (5), and by noting that $\boldsymbol{\nu}=\frac{\operatorname{dev} \boldsymbol{\tau}}{\|\operatorname{dev} \boldsymbol{\tau}\|}$ where $\operatorname{dev}(\cdot)$ stands for the deviatoric part, the model of Example 3.3 can be written in the following format

$$
\begin{gathered}
\mathbf{d}=\mathbf{d}_{\mathbf{e}}+\mathbf{d}_{\mathbf{p}}, \\
\mathbf{d}_{\mathbf{e}}=\frac{\partial^{2} \sigma}{\partial \boldsymbol{\tau} \partial \boldsymbol{\tau}}: \hat{\boldsymbol{\tau}}^{\log }, \\
\mathbf{d}_{\mathbf{p}}=\frac{\langle f\rangle}{(K+H) \beta+R(\beta-f)} \frac{\operatorname{dev} \boldsymbol{\tau}}{\|\operatorname{dev} \boldsymbol{\tau}\|}\left\langle\frac{\operatorname{dev} \boldsymbol{\tau}}{\|\operatorname{dev} \boldsymbol{\tau}\|}: \hat{\boldsymbol{\tau}}^{\log }\right\rangle, \\
\dot{a}=\frac{\langle f\rangle}{(K+H) \beta+R(\beta-f)}\left\langle\frac{\operatorname{dev} \boldsymbol{\tau}}{\|\operatorname{dev} \boldsymbol{\tau}\|}: \hat{\boldsymbol{\tau}}^{\log }\right\rangle, \\
\hat{\mathbf{q}}^{\log }=\frac{2}{3} H \mathbf{d}_{\mathbf{p}} .
\end{gathered}
$$

The particular case in which $\beta=0$ and the material state is constrained to lie on the yield surface defined by $f=0$ corresponds to classical plasticity Lubliner et al. 1993. Another particular case of interest arises where $R=0$. Then upon taking the limit as $\beta \rightarrow 0$ and by involving the consistency condition $\dot{f}=0$, it can be proved Panoskaltsis et al. 2011] that the material model has as a limit the standard linear elastic-plastic model.

Remark 3.7. It is instructive to turn back in the original approach introduced in Xiao et al. 1997 and exploit the formulation of the governing equations by adopting the alias point of view. If this is the case Eqs. (17), (21) and (22) can be interpreted as the basic equations of the theory as perceived in the rotating frame, while Eqs. (11), 30 and (31) are their counterparts in the fixed background frame. Note that both triplets of equations are essentially of the same format, with the basic difference between them relying in the presence of some extra terms in Eqs. (30), (31). These terms have no apparent source in identifiable physical sources, in 
particular matter; they just appear due to the relative spin $\left(\boldsymbol{\Omega}^{\log }\right)$ between the two frames.

Remark 3.8. Note that the present formulation, which is based on choosing a preferred configuration where the governing equations take their simplest form and interpreting the basic kinematical quantity as a Lie derivative, has its origins in the covariant approach to generalized plasticity as it is discussed in Panoskaltsis et al. 2008b; (see also Panoskaltsis et al. 2011]). Nevertheless, the present formulation is not a covariant one, since it employs the logarithmic rate which is invariant only with respect to arbitrary superposed spatial rigid motions (isometries) and not with respect to arbitrary spatial diffeomorphisms, as required by the spatial covariance concept, (see, e.g., Marsden and Hughes, 1994, pp. 99-102]). Accordingly, we term the present formulation as material frame-indifferent.

\section{Computational aspects}

In this section the numerical implementation of a generalized plasticity model within the context of the logarithmic rate is presented. The implementation procedure may in principle be formulated equivalently with respect to the $\mathbf{R}^{\log }$-rotated or the spatial configuration. Since the theory presented herein considers the case of large scale plastic flow, the kinematics of the problem, together with the principle of spatial covariance (see Remark 3.8, suggest that a numerical formulation in terms of the Kirchhoff stress and its logarithmic derivative is more fundamental. Moreover, the spatial approach has an additional advantage since it leads to an algorithm which falls within the context of the well-known incrementally objective algorithms (see, e.g., the related discussion in Simo and Hughes, 1998, pp. 276278]; see also Hughes and Winget [1980], Rubinstein and Atluri [1983], Pinsky et al. 1983]).

As a first step the governing equation of the formulation, (i.e. Eqs. (1), (6), (30) and (31) are rephrased in a format which is well suited for computational use. This is achieved by substituting from Eqs. (6) and (30) into Eq. (1), so that the following relations are retrieved

$$
\begin{gathered}
\mathbf{d}=\frac{\partial^{2} \sigma}{\partial \boldsymbol{\tau} \partial \boldsymbol{\tau}}: \hat{\boldsymbol{\tau}}^{\log }+h \boldsymbol{\lambda}\left(\boldsymbol{\tau}, \mathbf{a}, \mathbf{R}^{\log }\right)\left\langle\boldsymbol{\nu}: \hat{\boldsymbol{\tau}}^{\log }\right\rangle, \\
\hat{\mathbf{a}}^{\log }=h \boldsymbol{\mu}\left(\boldsymbol{\tau}, \mathbf{a}, \mathbf{R}^{\log }\right)\left\langle\boldsymbol{\nu}: \hat{\boldsymbol{\tau}}^{\log }\right\rangle .
\end{gathered}
$$

For known rate of deformation d, Eqs. (36) and (37), form a system of two equations with respect to $\boldsymbol{\tau}$ and $\mathbf{a}$. This system can be solved upon time discretization within the framework of a predictor-corrector algorithm Panoskaltsis et al. $2008 \mathrm{~b}$ a. The details of the implementation procedure follow. 
Let $\mathrm{J} \in[0, T]$ be the time interval of interest. It is assumed that at time $t_{\mathrm{n}} \in \mathrm{J}$, the configuration of the body of interest $\omega_{\mathrm{n}}=\varphi_{\mathrm{n}}(\boldsymbol{\Omega})$, i.e. $\omega_{\mathrm{n}} \equiv\left\{\mathbf{x}_{\mathrm{n}}=\mathbf{x}_{\mathrm{n}}(\mathbf{X}) / \mathbf{X} \in \boldsymbol{\Omega}\right\}$, along with the state variables $\left\{\boldsymbol{\tau}_{\mathrm{n}}, \mathbf{a}_{\mathrm{n}}\right\}$, are known.

Assume a time increment $\Delta t_{\mathrm{n}}$, which drives the time to $t_{\mathrm{n}+1}$ and the body configuration to

$$
\omega_{\mathrm{n}+1} \equiv\left\{\mathbf{x}_{\mathrm{n}+1}=\mathbf{x}_{\mathrm{n}+1}(\mathbf{X}) / \mathbf{X} \in \mathbf{\Omega}\right\}
$$

where

$$
\mathbf{x}_{\mathrm{n}+1}(\mathbf{X})=\mathbf{x}_{\mathrm{n}}(\mathbf{X})+\mathbf{u}\left(\mathbf{x}_{\mathrm{n}}(\mathbf{X})\right)
$$

and $\mathbf{u}$ stands for the incremental displacement field which is assumed to be given.

The corresponding deformation gradient reads

$$
\mathbf{F}_{\mathrm{n}+1}(\mathbf{X})=\frac{\partial \mathbf{x}_{\mathrm{n}+1}}{\partial \mathbf{X}}
$$

Then the algorithmic problem at hand is to update the stress tensor and the internal variable vector to the time step $t_{\mathrm{n}+1}$ in a manner consistent with the (time continuous) Eqs. (36), (37). To this end the continuous equations will be time discretized by the backward Euler scheme which is first order accurate and unconditionally stable. Because of the presence of the logarithmic rates within the continuous equations, algorithmic approximations for these objects are derived on the basis of our interpretation of the logarithmic rate as a Lie derivative. In order to accomplish this goal we exploit Eq. (29) which at the $\omega_{\mathrm{n}+1}$ configuration reads

$$
\hat{\mathbf{a}}_{\mathrm{n}+1}^{\log }=L_{\mathbf{w}^{\log }}(\mathbf{a})_{\mathrm{n}+1}=\mathbf{x}_{\mathrm{n}+1_{*}}\left[\frac{d}{d t} \mathbf{x}_{\mathrm{n}+1}^{*}\left(\mathbf{a}_{\mathrm{n}+1}\right)\right] \text {. }
$$

By performing a pull-back operation, Eq. (38) can be written consecutively as

$$
\mathbf{x}_{\mathrm{n}+1}^{*}\left(\hat{\mathbf{a}}_{\mathrm{n}+1}^{\log }\right)=\frac{\partial}{\partial t}\left[\mathbf{x}_{\mathrm{n}+1}^{*}\left(\mathbf{a}_{\mathrm{n}+1}\right)\right]=\dot{\mathbf{a}}_{\mathrm{n}+1}^{\prime}=\frac{1}{\Delta t_{\mathrm{n}}}\left(\mathbf{a}_{\mathrm{n}+1}^{\prime}-\mathbf{a}_{\mathrm{n}}^{\prime}\right)
$$

which in turn may be written in component form as

$$
\begin{aligned}
& \left(R_{j_{1} i_{1}}^{\log }\right)_{n+1} \ldots\left(R_{j_{m} i_{m}}^{\log }\right)_{n+1}\left(\hat{a}_{i_{1} \ldots i_{m}}^{\log }\right)_{n+1}=\frac{1}{\Delta t_{n}}\left[\left(a_{j_{1} \ldots j_{m}}^{\prime}\right)_{n+1}-\left(a_{j_{1} \ldots j_{m}}^{\prime}\right)_{n}\right]= \\
& \frac{1}{\Delta t_{n}}\left[\left(R_{j_{1} i_{1}}^{\log }\right)_{n+1} \ldots\left(R_{j_{m} i_{m}}^{\log }\right)_{n+1}\left(a_{i_{1} \ldots i_{m}}\right)_{n+1}-\left(R_{j_{1} i_{1}}^{\log }\right)_{n} \ldots\left(R_{j_{m} i_{m}}^{\log }\right)_{n}\left(a_{i_{1} \ldots i_{m}}\right)_{n}\right]
\end{aligned}
$$

from which $\left(\hat{\mathrm{a}}_{i_{1} \ldots i_{\mathrm{m}}}^{\log }\right)_{\mathrm{n}+1}$ can be determined as

$$
\begin{aligned}
& \left(\hat{a}_{i_{1} \ldots i_{m}}^{\log }\right)_{n+1}=\frac{1}{\Delta t_{n}}\left[\left(a_{i_{1} \ldots i_{m}}\right)_{n+1}-\left(R_{i_{1} j_{1}}^{\log }\right)_{n+1}\left(R_{j_{1} l_{1}}^{\log }\right)_{n} \ldots\left(R_{i_{m} j_{m}}^{\log }\right)_{n+1}\left(R_{j_{m} l_{m}}^{\log }\right)_{n}\left(a_{l_{1} \ldots l_{m}}\right)_{n}\right]= \\
& \frac{1}{\Delta t_{n}}\left[\left(a_{i_{1} \ldots i_{m}}\right)_{n+1}-\left(r_{i_{1} l_{1}}^{\log }\right)_{n+1} \ldots\left(r_{i_{m} l_{m}}^{\log }\right)_{n+1}\left(a_{l_{1} \ldots l_{m}}\right)_{n}\right]
\end{aligned}
$$


that is

$$
\hat{\mathbf{a}}_{\mathrm{n}+1}^{\log }=\frac{1}{\Delta t_{\mathrm{n}}} \mathbf{g}\left(\mathbf{a}_{\mathrm{n}+1}, \mathbf{a}_{\mathrm{n}}, \mathbf{r}_{\mathrm{n}+1}^{\log }\right),
$$

where $\mathbf{g}$ is a (tensorial) function of the denoted arguments, where the (orthogonal) tensor $\mathbf{r}_{\mathrm{n}+1}^{\log }: \mathrm{T}_{\mathbf{x}_{\mathrm{n}+1}} \mathbf{E}^{3} \times \mathrm{T}_{\mathbf{x}_{\mathrm{n}+1}} \mathbf{E}^{3} \rightarrow, \mathbf{r}_{\mathrm{n}+1}^{\log }=\mathbf{R}_{\mathrm{n}+1}^{\log \mathbf{T}} \mathbf{R}_{\mathrm{n}}^{\log }$, with components

$$
\left(r_{i_{\mathrm{k}} l_{\mathrm{k}}}^{\log }\right)_{\mathrm{n}+1}=\left(R_{i_{\mathrm{k}} j_{\mathrm{k}}}^{\log }\right)_{\mathrm{n}+1}\left(R_{j_{\mathrm{k}} l_{\mathrm{k}}}^{\log }\right)_{\mathrm{n}},
$$

is defined to be as the relative $\mathbf{R}^{\log }$-rotation tensor with respect to the configuration $\omega_{\mathrm{n}+1}$. By means of Eq. (39) an algorithmic approximation to the logarithmic rate of the 2-rank Kirchhoff stress tensor can be found to be

$$
\hat{\boldsymbol{\tau}}_{\mathrm{n}+1}^{\log }=\frac{1}{\Delta t_{\mathrm{n}}}\left(\boldsymbol{\tau}_{\mathrm{n}+1}-\mathbf{r}_{\mathrm{n}+1} \boldsymbol{\tau}_{\mathrm{n}} \mathbf{r}_{\mathrm{n}+1}^{\mathbf{T}}\right)
$$

We note also the presence of the rate of deformation tensor $\mathbf{d}$ within the basic equations. An algorithmic approximation to $\mathbf{d}$ can be found in a manner identical with the one for the approximation of the logarithmic rate Simo and Hughes, 1998, pp. 282-285]; Panoskaltsis et al., 2008a this approximation reads

$$
\mathbf{d}_{\mathrm{n}+1}=\frac{1}{2 \Delta t_{\mathrm{n}}}\left[\mathbf{i}-\left(\mathbf{f}_{\mathrm{n}+1} \mathbf{f}_{\mathrm{n}+1}^{\mathbf{T}}\right)^{-1}\right]
$$

where $\mathbf{f}_{\mathrm{n}+1}$ is the relative deformation gradient, which is defined to be $\mathbf{f}_{\mathrm{n}+1}=$ $\mathbf{F}_{\mathrm{n}+1} \mathbf{F}_{\mathrm{n}}^{-1}$.

On the basis of these developments the time discrete counterparts of Eqs. (36) and (34) read

$$
\begin{aligned}
& \frac{1}{2 \Delta t_{\mathrm{n}}}\left[\mathbf{i}-\left(\mathbf{f}_{\mathrm{n}+1} \mathbf{f}_{\mathrm{n}+1}^{\mathbf{T}}\right)^{-1}\right]=\frac{1}{\Delta t_{\mathrm{n}}}\left[\frac{\partial^{2} \sigma\left(\boldsymbol{\tau}_{\mathrm{n}+1}\right)}{\partial \boldsymbol{\tau}_{\mathrm{n}+1} \partial \boldsymbol{\tau}_{\mathrm{n}+1}}:\left(\boldsymbol{\tau}_{\mathrm{n}+1}-\mathbf{r}_{\mathrm{n}+1}^{\log } \boldsymbol{\tau}_{\mathrm{n}} \mathbf{r}_{\mathrm{n}+1}^{\log \mathbf{T}}\right)\right. \\
& \left.+h_{\mathrm{n}+1} \lambda\left(\boldsymbol{\tau}_{\mathrm{n}+1}, \mathbf{a}_{\mathrm{n}+1}, \mathbf{R}_{\mathrm{n}+1}^{\log }\right)\left\langle\boldsymbol{\nu}_{\mathrm{n}+1}:\left(\boldsymbol{\tau}_{\mathrm{n}+1}-\mathbf{r}_{\mathrm{n}+1}^{\log } \boldsymbol{\tau}_{\mathrm{n}} \mathbf{r}_{\mathrm{n}+1}^{\log \mathbf{T}}\right)\right\rangle\right]
\end{aligned}
$$

and

$$
\begin{aligned}
& \frac{1}{\Delta t_{\mathrm{n}}} \mathbf{g}\left(\mathbf{a}_{\mathrm{n}+1}, \mathbf{a}_{\mathrm{n}}, \mathbf{r}_{\mathrm{n}+1}^{\log }\right)= \\
& \frac{1}{\Delta t_{\mathrm{n}}} h_{\mathrm{n}+1} \boldsymbol{\mu}\left(\boldsymbol{\tau}_{\mathrm{n}+1}, \mathbf{a}_{\mathrm{n}+1}, \mathbf{R}_{\mathrm{n}+1}^{\log }\right)\left\langle\boldsymbol{\nu}_{\mathrm{n}+1}:\left(\boldsymbol{\tau}_{\mathrm{n}+1}-\mathbf{r}_{\mathrm{n}+1}^{\log } \boldsymbol{\tau}_{\mathrm{n}} \mathbf{r}_{\mathrm{n}+1}^{\log \mathbf{T}}\right)\right\rangle
\end{aligned}
$$

respectively, where the quantities $h_{\mathrm{n}+1}=h_{\mathrm{n}+1}\left(\boldsymbol{\tau}_{\mathrm{n}+1}, \mathbf{a}_{\mathrm{n}+1}, \mathbf{R}_{\mathrm{n}+1}^{\log }\right)$ and $\boldsymbol{\nu}_{\mathrm{n}+1}=$ $\frac{\partial f}{\partial \boldsymbol{\tau}}\left(\boldsymbol{\tau}_{\mathrm{n}+1}, \mathbf{a}_{\mathrm{n}+1}, \mathbf{R}_{\mathrm{n}+1}^{\log }\right)$, in which $f=f\left(\boldsymbol{\tau}_{\mathrm{n}+1}, \mathbf{a}_{\mathrm{n}+1}, \mathbf{R}_{\mathrm{n}+1}^{\log }\right)=$ const. is the time discrete expression for the loading surfaces, can be all expressed in terms of the basic variables. The details of the solution procedure of the system of Eqs. 40, , 41, can be found in Panoskaltsis et al. 2008a.

Remark 4.1. It is emphasized that the functions $\boldsymbol{\lambda}, \boldsymbol{\mu}$ and constitute the pushforwards of the functions $\boldsymbol{\lambda}^{\prime}, \boldsymbol{\mu}^{\prime}$ and $\boldsymbol{\nu}^{\prime}$ in the spatial configuration. Accordingly, 
the dependence of these functions on $\mathbf{R}^{\log }$ cannot be arbitrary, since it must be consistent with the corresponding push-forward operation.

Remark 4.2. The logarithmic spin which in fact defines the logarithmic rotation matrix can be found by means of the time discrete counterpart of Eq. (4), by noting that

(i) The spin tensor $\mathbf{w}_{\mathrm{n}+1}$ at the time step $t_{\mathrm{n}+1}$ in terms of the (time discrete) velocity gradient $\mathbf{l}_{\mathrm{n}+1}=\left(\mathbf{F}_{\mathrm{n}+1}-\mathbf{F}_{\mathrm{n}}\right) \mathbf{F}_{\mathrm{n}+1}^{-1}$ reads

$$
\mathbf{w}_{\mathrm{n}+1}=\frac{1}{2 \Delta t_{\mathrm{n}}}\left(\mathbf{l}_{\mathrm{n}+1}-\mathbf{l}_{\mathrm{n}+1}^{\mathbf{T}}\right),
$$

(ii) The left Cauchy-Green tensor $\mathbf{b}_{\mathrm{n}+1}$ at the time step $t_{\mathrm{n}+1}$ is defined in terms of the deformation gradient $\mathbf{F}_{\mathrm{n}+1}$ as $\mathbf{b}_{\mathrm{n}+1}=\mathbf{F}_{\mathrm{n}+1} \mathbf{F}_{\mathrm{n}+1}^{\mathbf{T}}$.

A closed form solution for determining the eigenvalues of $\mathbf{b}_{\mathrm{n}+1}$ can be found in Bruhns et al. 1999. This solution is also used in the framework introduced in the present work. The evaluation of the left Cauchy-Green tensor introduces an additional complexity within a finite element computational framework. However, the advantages of the logarithmic spin concept for the simulation of large strain processes render such an approach highly advantageous in terms of simulation fidelity Xiao et al. 2001; Balieu et al., 2013. Furthermore, the numerical implementation of the method within a generalized plasticity - thus yield surface-free setting - results in computational gains pertaining to the absence of a constrain equation as described in Section 4 of this paper.

Remark 4.3. The most critical step in the algorithmic procedure is the determination of the logarithmic rotation $\mathbf{R}_{n+1}^{\log }$ by means of the exponential mapping (see Eqs. (18), (19) whenever the logarithmic spin $\boldsymbol{\Omega}_{\mathrm{n}+1}^{\log }$ is known. There are several approaches for computing the exponential map; see for instance the classical paper by Moler and Loan 2003. Within the contemporary literature of continuum mechanics the exponential of matrix is usually computed either by means of the so-called Rodrigues formula (see, e.g., Simo and Hughes, 1998, p. 295]), or by the quaternion approach (see further in [Simo and Hughes, 1998, pp. 296-297]. A formal comparison between these two approaches, where special emphasis is paid to computer graphics, can be found in Grassia [1998. Further information can also be found in Stuelpnagel 1964.

Remark 4.4. It is reminded that generalized plasticity does not employ the yield surface concept as a basic ingredient. Thus, unlike the classical elastic-plastic case, the basic variables $(\boldsymbol{\tau}, \mathbf{a})$ are no longer constrained to lie within the closure of this elastic domain; accordingly, unlike the classic elastic-plastic case where the evolution equations define a unilaterally constrained problem of evolution, (see, e.g., Simo and Hughes, 1998, pp. 273-275, 293]) in the present case the evolution equations 
just form the differential system of Eqs. 40 and 41. Due to this fundamental difference, the consistency condition and accordingly the consistency parameter are absent from the model governing equations. From an algorithmic point of view this absence results in a simpler algorithm and more computer power is preserved.

Remark 4.5. It is interesting to note that the particular case of a quasi-yield surface based model - recall Example 3.4 - offers an additional computational advantage. More specifically, solution of the aforementioned system in general - see, e.g., Panoskaltsis et al., 2008a - is pursued by an (elastic) predictor-(plastic) corrector algorithm. Thus, an elastic solution is sought in the predictor phase upon freezing the plastic flow. This check is based on the time-discrete loading unloadingconditions, i.e. on examining whether the material state is elastic or plastic and also whether elastic or plastic loading takes place. For a quasi-yield surface model, since there are no elastic states the first check is entirely bypassed.

Example 4.1. If we further assume that the potential $\sigma$ has the following particular form Bruhns et al. 1999

$$
2 \sigma=\frac{1+\nu}{\mathrm{E}}:\|\boldsymbol{\tau}\|^{2}-\frac{\nu}{\mathrm{E}}(\operatorname{tr} \boldsymbol{\tau})^{2},
$$

where $\mathrm{E}$ is the Young modulus and $\nu$ is the Poisson ratio, then the hyperelastic rate stress-deformation relations (5) reads

$$
\mathbf{d}_{\mathbf{e}}=\frac{1+\nu}{\mathrm{E}} \hat{\boldsymbol{\tau}}^{\log }-\frac{\nu}{\mathrm{E}} \operatorname{tr} \dot{\boldsymbol{\tau}} \mathbf{i}
$$

The corresponding time discrete counterpart of the model reads

$$
\begin{aligned}
& \frac{1}{2}\left[\mathbf{i}-\left(\mathbf{f}_{\mathrm{n}+1} \mathbf{f}_{\mathrm{n}+1}^{\mathbf{T}}\right)^{-1}\right]=\left[\frac{1+\nu}{\mathrm{E}}\left(\boldsymbol{\tau}_{\mathrm{n}+1}-\mathbf{r}_{\mathrm{n}+1}^{\log } \boldsymbol{\tau}_{\mathrm{n}} \mathbf{r}_{\mathrm{n}+1}^{\log \mathbf{T}}\right)-\frac{\nu}{\mathrm{E}} \operatorname{tr}\left(\boldsymbol{\tau}_{\mathrm{n}+1}-\boldsymbol{\tau}_{\mathrm{n}}\right) \mathbf{i}\right. \\
& +\frac{\left\langle f_{n+1}\right\rangle}{(K+H) \beta+R(\beta-f)} \frac{\operatorname{dev} \boldsymbol{\tau}_{\mathrm{n}+1}}{\left\|\operatorname{dev} \boldsymbol{\tau}_{\mathrm{n}+1}\right\|}\left\langle\frac{\operatorname{dev} \boldsymbol{\tau}_{\mathrm{n}+1}}{\left\|\operatorname{dev} \boldsymbol{\tau}_{\mathrm{n}+1}\right\|}:\left(\boldsymbol{\tau}_{\mathrm{n}+1}-\mathbf{r}_{\mathrm{n}+1}^{\log } \boldsymbol{\tau}_{\mathrm{n}} \mathbf{r}_{\mathrm{n}+1} \log \mathbf{T}\right)\right\rangle, \\
& a_{\mathrm{n}+1}-a_{\mathrm{n}}=\frac{\left\langle f_{n+1}\right\rangle}{(K+H) \beta+R(\beta-f)}\left\langle\frac{\operatorname{dev} \boldsymbol{\tau}_{\mathrm{n}+1}}{\left\|\operatorname{dev} \boldsymbol{\tau}_{\mathrm{n}+1}\right\|}:\left(\boldsymbol{\tau}_{\mathrm{n}+1}-\mathbf{r}_{\mathrm{n}+1}^{\log } \boldsymbol{\tau}_{\mathrm{n}} \mathbf{r}_{\mathrm{n}+1}^{\log \mathbf{T}}\right)\right\rangle, \\
& \left(\mathbf{q}_{\mathrm{n}+1}-\mathbf{r}_{\mathrm{n}+1}^{\log } \mathbf{q}_{\mathrm{n}} \mathbf{r}_{\mathrm{n}+1}^{\log \mathbf{T}}\right)= \\
& \frac{2}{3} H \frac{\left\langle f_{n+1}\right\rangle}{(K+H) \beta+R(\beta-f)} \frac{\operatorname{dev} \boldsymbol{\tau}_{\mathrm{n}+1}}{\left\|\operatorname{dev} \boldsymbol{\tau}_{\mathrm{n}+1}\right\|}\left\langle\frac{\operatorname{dev} \boldsymbol{\tau}_{\mathrm{n}+1}}{\left\|\operatorname{dev} \boldsymbol{\tau}_{\mathrm{n}+1}\right\|}:\left(\boldsymbol{\tau}_{\mathrm{n}+1}-\mathbf{r}_{\mathrm{n}+1}^{\log \boldsymbol{\tau}_{\mathrm{n}} \mathbf{r}_{\mathrm{n}+1} \log \mathbf{T}}\right)\right\rangle
\end{aligned}
$$

where $f_{n+1}$ stands for the time discrete counterpart of the expression for the loading surfaces, that is

$$
f_{n+1}=f\left(\boldsymbol{\tau}_{n+1}, a_{n+1}, \mathbf{q}_{n+1}\right)=\left\|\boldsymbol{\tau}_{n+1}-\mathbf{q}_{n+1}\right\|-\sqrt{\frac{2}{3}}\left(\sigma_{y}+K a_{n+1}\right)=\text { const. }
$$


Note that in this particular case, Eqs. 42, 43) and (44) constitute a system of three equations in the three unknowns $\boldsymbol{\tau}_{n+1}, a_{n+1}$ and $\mathbf{q}_{n+1}$.

\section{Numerical simulations}

As a final step, we check the performance of the proposed algorithmic scheme by implementing numerically the material model discussed in Examples $3.1,3.2$ and 4.1. We consider two problems of large scale plastic flow, namely a simple shear test and an additional test in which the material is subjected to a smooth strain cycle. In both tests the deformation can be assumed as homogeneous so that the model will be implemented within a standard MATLAB environment.

The model parameters which are used in both tests are those considered in Lubliner et al. [1993, that is

$$
\begin{array}{llll}
E=300 \quad v=0.30 \quad \sigma_{y}=10 & \beta=5.00 & R=30.00 \\
\text { Kinematic Hardening Only: } & H=E / 10 &
\end{array}
$$

\subsection{Simple shear}

The simple shear problem is a classical one within the context of large deformation plasticity (see, e.g., Nagtegaal and De Jong 1982, Dafalias 1983, Atluri 1984. Bruhns et al. [1999, Liu and Hong 2001, Eshraghi et al. [2013 and also the theoretical developments given in Liu [2004,, Liu and Hong [2001], Cheviakov et al. 2013]) and is defined as

$$
x_{1}=X_{1}+\gamma X_{2}, x_{2}=X_{2}, x_{3}=X_{3}
$$

where $\gamma$ is the shearing parameter.

The predictions of the model for three different values of the kinematic hardening modulus are shown in Fig. 1 and Fig. 2 for the shear $\boldsymbol{\tau}_{12}$ and normal $\boldsymbol{\tau}_{11}$ stress components respectively. By referring to these figures we note that the model predicts monotonic stress-strain curves for both the cases of a strain hardening and an elastic-perfectly plastic material. Moreover, the unique ability of the present model in predicting strain softening response for negative values of hardening modulus (in this case $H=-15$ ) is verified Lubliner et al., 1993.

The predictions of the model for the particular cases where $R=0$, which corresponds to the simple model presented in Lubliner 1991, and $\beta=0$ which is the limiting case corresponding to classical plasticity are also shown, in Fig. 3 and Fig. 4. respectively. The linear stress-strain relation predicted by the model for $\beta=0$ is noteworthy. 


\subsection{Response under a strain cycle}

As a second application, we consider a problem which has been used as an identification problem for the logarithmic rate within the context of a (hypo)elastic formulation [Meyers et al. 2003]. A similar problem has been considered also within the context of an elastic - viscoplastic material in the very recent paper by Jabareen 2015. More precisely a square material element of size $H \times H$ is imposed onto a strain cycle where both upper corners rotate along a cycle with radius. The straining is applied in a way such that the element is subjected to combined extension in $X_{2}$ - direction and shear in the $X_{1}-X_{2}$-direction but remains parallelogram in shape (see Figure 1 in Meyers et al. 2003). This deformation process is defined as

$$
\begin{aligned}
& x_{1}=X_{1}+\frac{\sin \varphi \frac{r}{H}}{1+(1-\cos \varphi) \frac{r}{H}} X_{2}, \\
& x_{2}=1+(1-\cos \varphi) \frac{r}{H} X_{2}, \\
& x_{3}=X_{3},
\end{aligned}
$$

where $\varphi$ is the rotation angle.

The stress curves for the elastic case and for a relatively high value of the ratio $\frac{r}{H}$, are shown in Fig. 5 the corresponding elastic-plastic curves predicted the model and the back stresses are shown in Fig. 6 and Fig. 7 respectively. By comparing these results, we note that the elastic-plastic stress curves have the same qualitative characteristics with the elastic ones; nevertheless, due to the dissipative properties of the material, the resulting stresses do not vanish at the end of the cycle and a stress ratcheting effect appears.

This ratchetting effect is better illustrated in the case of repeated cyclic loading as shown in Fig. 8 and Fig. 9 where seven cycles of imposed deformation are shown. In Fig. 8 the stress components for a generalized plasticity case with a value $R=$ 30.00 are shown. In Fig. 9 the limiting case resulting for $\beta=0$ is also presented. The plots show the expected results for a generalized plasticity based model Lubliner, 1975, Auricchio and Taylor, 1995], i.e. the stress curves exhibit a ratcheting effect which is stabilized after a few cycles.

\section{Concluding remarks}

The main thrust of this paper is the presentation of a spatial (Eulerian) version of the theory of rate independent generalized plasticity, which is based on the rather newly discovered concept of the logarithmic rate. In particular in this paper:

(i) Based on the logarithmic spin and the corresponding logarithmic rotation we have introduced a rotationally neutralized configuration which is unaffected by spatial rigid body motions, so that the fulfillment of the material frameindifference principle is automatically ensured.

(ii) We have developed the theory in the rotationally neutralized configuration. In particular we have shown that the structure of the theory in this configuration 
is essentially identical to that of the infinitesimal theory.

(iii) We have derived the equivalent Eulerian version of the theory by applying a (standard) push-forward operation.

Moreover, in the course of the development of the theory we have identified the logarithmic rate by a Lie derivative. Building on this we have proposed an incrementally objective algorithm for the numerical implementation of generalized plasticity based models. We have also tested the performance of the algorithm by two representative numerical examples.

\section{References}

Abraham, R., Marsden, J. E. and Ratiu, T. [1988] Manifolds, Tensor Analysis, and Applications, Applied Mathematical Sciences, Vol. 75 (Springer, New York).

Atluri, S. N. [1984] "On constitutive relations at finite strain: Hypo-elasticity and elastoplasticity with isotropic or kinematic hardening," Computer Methods in Applied Mechanics and Engineering 43(2), 137-171.

Auricchio, F. and Taylor, R. L. [1995] "Two material models for cyclic plasticity: Nonlinear kinematic hardening and generalized plasticity," International Journal of Plasticity $\mathbf{1 1}(1), 65-98$.

Balieu, R., Lauro, F., Bennani, B., Delille, R., Matsumoto, T. and Mottola, E. [2013] "A fully coupled elastoviscoplastic damage model at finite strains for mineral filled semi-crystalline polymer," International Journal of Plasticity 51, 241 - 270, doi: http://dx.doi.org/10.1016/j.ijplas.2013.05.002.

Bernstein, B. [1960] "Hypo-elasticity and elasticity," Archive for Rational Mechanics and Analysis 6(1), 89-104.

Bishop, R. L. and Goldberg, S. I. [1980] Tesnor Analysis on Manifolds (Dover, New York).

Brepols, T., Vladimirov, I. N. and Reese, S. [2014] "Numerical comparison of isotropic hypo- and hyperelastic-based plasticity models with application to industrial forming processes," International Journal of Plasticity 63(0), 18-48.

Bruhns, O. T., Xiao, H. and Meyers, A. [1999] "Self-consistent eulerian rate type elastoplasticity models based upon the logarithmic stress rate," International Journal of Plasticity 15(5), 479-520.

Bruhns, O. T., Xiao, H. and Meyers, A. [2001] "Large simple shear and torsion problems in kinematic hardening elasto-plasticity with logarithmic rate," International Journal of Solids and Structures 38(4849), 8701-8722.

Cheviakov, A. F., Ganghoffer, J. F. and Rahouadj, R. [2013] "Finite strain plasticity models revealed by symmetries and integrating factors: The case of Dafalias spin model," International Journal of Plasticity 44(0), 47-67.

Dafalias, Y. F. [1983] "Corotational rates for kinematic hardening at large plastic deformations," Journal of Applied Mechanics 50(3), 561-565.

Dafalias, Y. F. [2011] "Objectivity in turbulence under change of reference frame and superposed rigid body motion," Journal of Engineering Mechanics 137(10), 699707.

Eshraghi, A., Jahed, H. and Papoulia, K. D. [2013] "Eulerian framework for inelasticity based on the Jaumann rate and a hyperelastic constitutive relation - Part II: Finite strain elastoplasticity," Journal of Applied Mechanics 80(2), 021028.

Frewer, M. [2009] "More clarity on the concept of material frame-indifference in classical continuum mechanics," Acta Mechanica 202(1-4), 213-246. 
Grassia, F. S. [1998] "Practical parameterization of rotations using the exponential map," J. Graph. Tools 3(3), 29-48.

Green, A. E. and McInnis, B. C. [1967] "Generalized Hypo-Elasticity," Proceedings of the Royal Society of Edinburgh, Section: A Mathematics 67(03), 220-230.

Hughes, T. J. R. and Winget, J. [1980] "Finite rotation effects in numerical integration of rate constitutive equations arising in large-deformation analysis," International Journal for Numerical Methods in Engineering 15(12), 1862-1867.

Jabareen, M. [2015] "Strongly objective numerical implementation and generalization of a unified large inelastic deformation model with a smooth elasticinelastic transition," International Journal of Engineering Science 96, 46 - 67, doi:http://dx.doi.org/10. 1016/j.ijengsci.2015.07.001.

Lehmann, T., Guo, Z. H. and Liang, H. Y. [1991] "The conjugacy between cauchy stress and logarithm of the left stretch tensor," European Journal of Mechanics a-Solids $\mathbf{1 0}(4), 395-404$.

Lin, R. [2002] "Numerical study of consistency of rate constitutive equations with elasticity at finite deformation," International Journal for Numerical Methods in Engineering 55(9), 1053-1077.

Lin, R. C., Schomburg, U. and Kletschkowski, T. [2003] "Analytical stress solutions of a closed deformation path with stretching and shearing using the hypoelastic formulations," European Journal of Mechanics - A/Solids 22(3), 443-461.

Liu, C.-S. [2004] "Lie symmetries of finite strain elasticperfectly plastic models and exactly consistent schemes for numerical integrations," International Journal of Solids and Structures 41(7), 1823-1853.

Liu, C.-S. and Hong, H.-K. [2001] "Using comparison theorem to compare corotational stress rates in the model of perfect elastoplasticity," International Journal of Solids and Structures 38(17), 2969-2987.

Liu, I. S. and Sampaio, R. [2014] "Remarks on material frame-indifference controversy," Acta Mechanica 225(2), 331-348.

Lubliner, J. [1974] "A simple theory of plasticity," International Journal of Solids and Structures 10(3), 313-319.

Lubliner, J. [1975] "On loading, yield and quasi-yield hypersurfaces in plasticity theory," International Journal of Solids and Structures 11(9), 1011-1016.

Lubliner, J. [1980] "An axiomatic model of rate-independent plasticity," International Journal of Solids and Structures 16(8), 709-713.

Lubliner, J. [1984] "A maximum-dissipation principle in generalized plasticity," Acta Mechanica 52(3-4), 225-237.

Lubliner, J. [1987] "Non-isothermal generalized plasticity," in H. Bui and Q. Nguyen (eds.), Thermomechanical Coupling in Solids, pp. 121-133.

Lubliner, J. [1991] "A simple model of generalized plasticity," International Journal of Solids and Structures 28(6), 769-778.

Lubliner, J., Taylor, R. L. and Auricchio, F. [1993] "A new model of generalized plasticity and its numerical implementation," International Journal of Solids and Structures 30(22), 3171-3184.

Marsden, J. E. and Hughes, T. J. R. [1994] Mathematical Foundations of Elasticity (Dover Publications, New York).

Meyers, A., Xiao, H. and Bruhns, O. T. [2003] "Elastic stress ratechetting and corotational stress rates," Technische Mechanik 23(2-4), 92-102.

Moler, C. and Loan, C. V. [2003] "Nineteen dubious ways to compute the exponential of a matrix, twenty-five years later," SIAM Review 45(1), 3-49.

Nagtegaal, J. and De Jong, J. [1982] "Some aspects of non-isotropic work-hardening in 
finite strain plasticity," Plasticity of metals at finite strain: theory, experiment and computation , 65-102.

Nemat-Nasser, S. [1982] "On finite deformation elasto-plasticity," International Journal of Solids and Structures 18(10), 857-872.

Noll, W. [1973] "Lectures on the foundations of continuum mechanics and thermodynamics," Archive for Rational Mechanics and Analysis 52(1), 62-92.

Panoskaltsis, V. P., Polymenakos, L. C. and Soldatos, D. [2008a] "Eulerian structure of generalized plasticity: Theoretical and computational aspects," Journal of Engineering Mechanics 134(5), 354-361.

Panoskaltsis, V. P., Polymenakos, L. C. and Soldatos, D. [2008b] "On large deformation generalized plasticity," Journal of Mechanics of Materials and Structures 3(3), 441457.

Panoskaltsis, V. P., Soldatos, D. and Triantafyllou, S. P. [2011] "The concept of physical metric in rate-independent generalized plasticity," Acta Mechanica 221(1-2), 49-64.

Pinsky, P. M., Ortiz, M. and Pister, K. S. [1983] "Numerical integration of rate constitutive equations in finite deformation analysis," Computer Methods in Applied Mechanics and Engineering 40(2), 137-158.

Reinhardt, W. D. and Dubey, R. N. [1996] "Coordinate-independent representation of spins in continuum mechanics," Journal of Elasticity 42(2), 133-144.

Rivlin, R. S. [1997] "Further remarks on the stress-deformation relations for isotropic materials," in G. Barenblatt and D. Joseph (eds.), Collected Papers of R.S. Rivlin, chap. 62 (Springer New York), pp. 1014-1035.

Rubinstein, R. and Atluri, S. N. [1983] "Objectivity of incremental constitutive relations over finite time steps in computational finite deformation analyses," Computer Methods in Applied Mechanics and Engineering 36(3), 277-290.

Shutov, A. and Ihlemann, J. [2014] "Analysis of some basic approaches to finite strain elasto-plasticity in view of reference change," International Journal of Plasticity 63, 183 - 197, doi:http://dx.doi.org/10.1016/j.ijplas.2014.07.004, deformation Tensors in Material Modeling in Honor of Prof. Otto T. Bruhns.

Simo, J. and Marsden, J. [1984a] "On the rotated stress tensor and the material version of the Doyle-Ericksen formula," Archive for Rational Mechanics and Analysis 86(3), 213-231.

Simo, J. and Pister, K. [1984] "Remarks on rate constitutive equations for finite deformation problems: computational implications," Computer Methods in Applied Mechanics and Engineering 46(2), $201-215$.

Simo, J. C. [1988] "A framework for finite strain elastoplasticity based on maximum plastic dissipation and the multiplicative decomposition: Part i. continuum formulation," Computer Methods in Applied Mechanics and Engineering 66(2), 199-219.

Simo, J. C. and Hughes, T. J. R. [1998] Computational Inelasticity (Springer-Verlag, New York).

Simo, J. C. and Marsden, J. E. [1984b] Stress tensors, Riemannian metrics and the alternative descriptions in elasticity, Lecture Notes in Physics, Vol. 195, chap. 21 (Springer Berlin Heidelberg), pp. 369-383.

Stuelpnagel, J. [1964] "On the parametrization of the three-dimensional rotation group," SIAM Review 6(4), 422-430.

Stumpf, H. and Hoppe, U. [1997] "The application of tensor algebra on manifolds to nonlinear continuum mechanics invited survey article," ZAMM - Journal of Applied Mathematics and Mechanics / Zeitschrift fr Angewandte Mathematik und Mechanik 77(5), 327-339.

Szekeres, P. [2004] A Course in Modern Mathematical Physics: Groups, Hilbert Space and 
Differential Geometry (Cambridge University Press, Cambridge).

Truesdell, C. [1955] "Hypo-elasticity," J. Rational Mech. Anal. 4. 83-133, 1019-1020.

Xiao, H., Bruhns, O. and Meyers, A. [2001] "Large strain responses of elastic-perfect plasticity and kinematic hardening plasticity with the logarithmic rate: Swift effect in torsion," International Journal of Plasticity 17(2), 211 - 235, doi:http://dx.doi. org/10.1016/S0749-6419(00)00054-1.

Xiao, H., Bruhns, O. and Meyers, A. [2014] "Free rate-independent elastoplastic equations," ZAMM - Journal of Applied Mathematics and Mechanics / Zeitschrift fr Angewandte Mathematik und Mechanik 94(6), 461-476, doi:10.1002/zamm. 201200260

Xiao, H., Bruhns, O. T. and Meyers, A. [1997] "Logarithmic strain, logarithmic spin and logarithmic rate," Acta Mechanica 124(1-4), 89-105.

Zhu, Y., Kang, G., Kan, Q. and Bruhns, O. T. [2014] "Logarithmic stress rate based constitutive model for cyclic loading in finite plasticity," International Journal of Plasticity 54(0), 34-55.

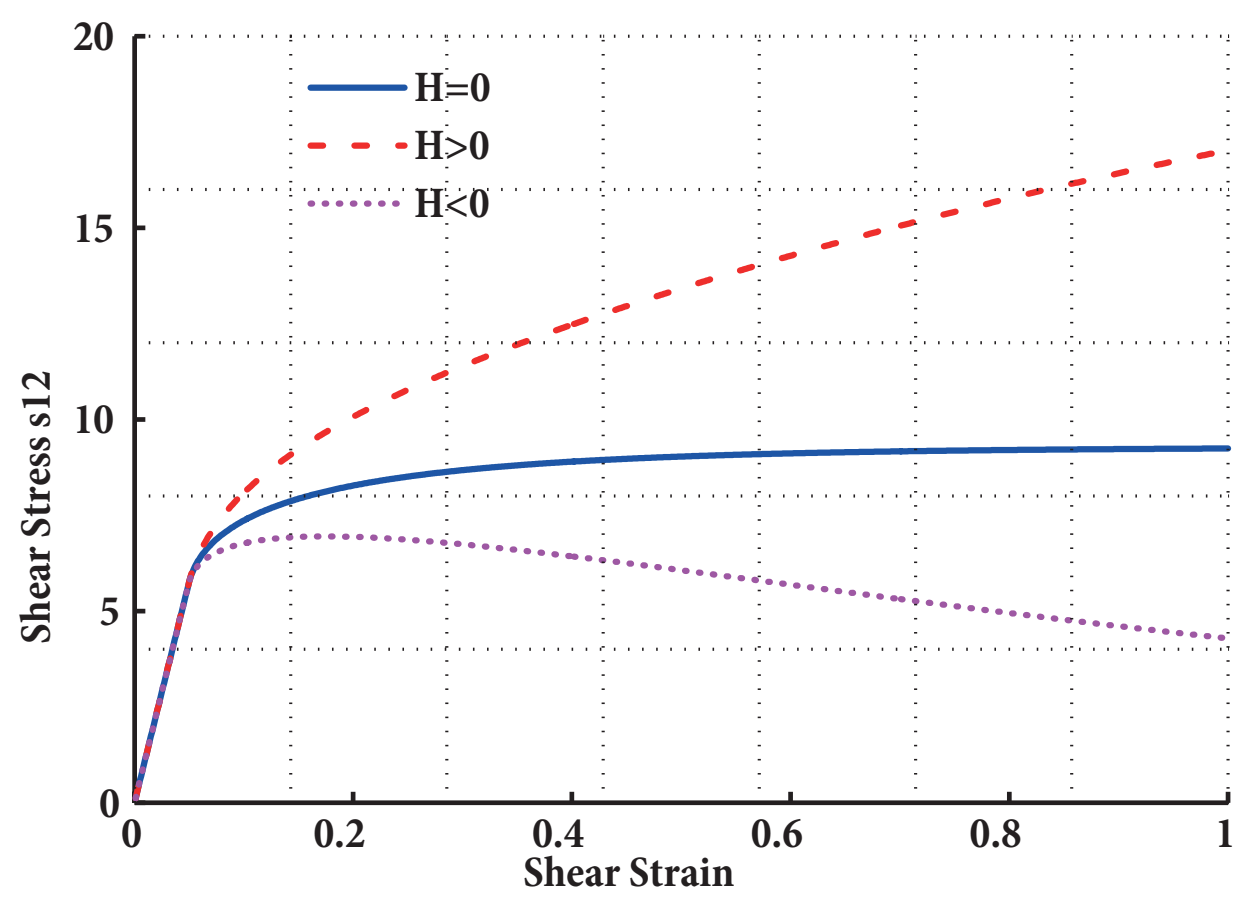

Fig. 1. Simple shear for different values of the kinematic hardening modulus H. Shear stress vs. shear strain 


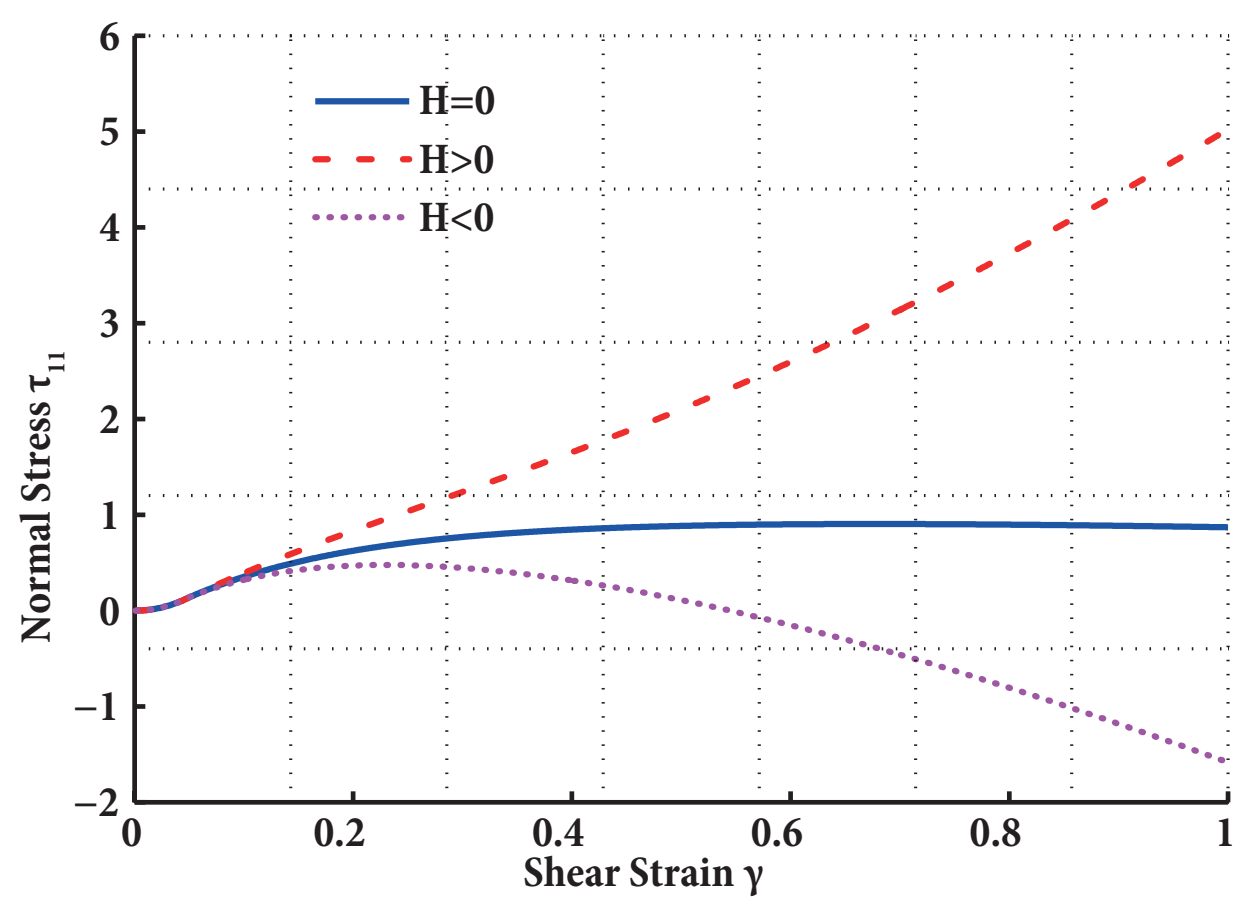

Fig. 2. Simple shear for different values of the kinematic hardening modulus H. Normal stress vs. shear strain 


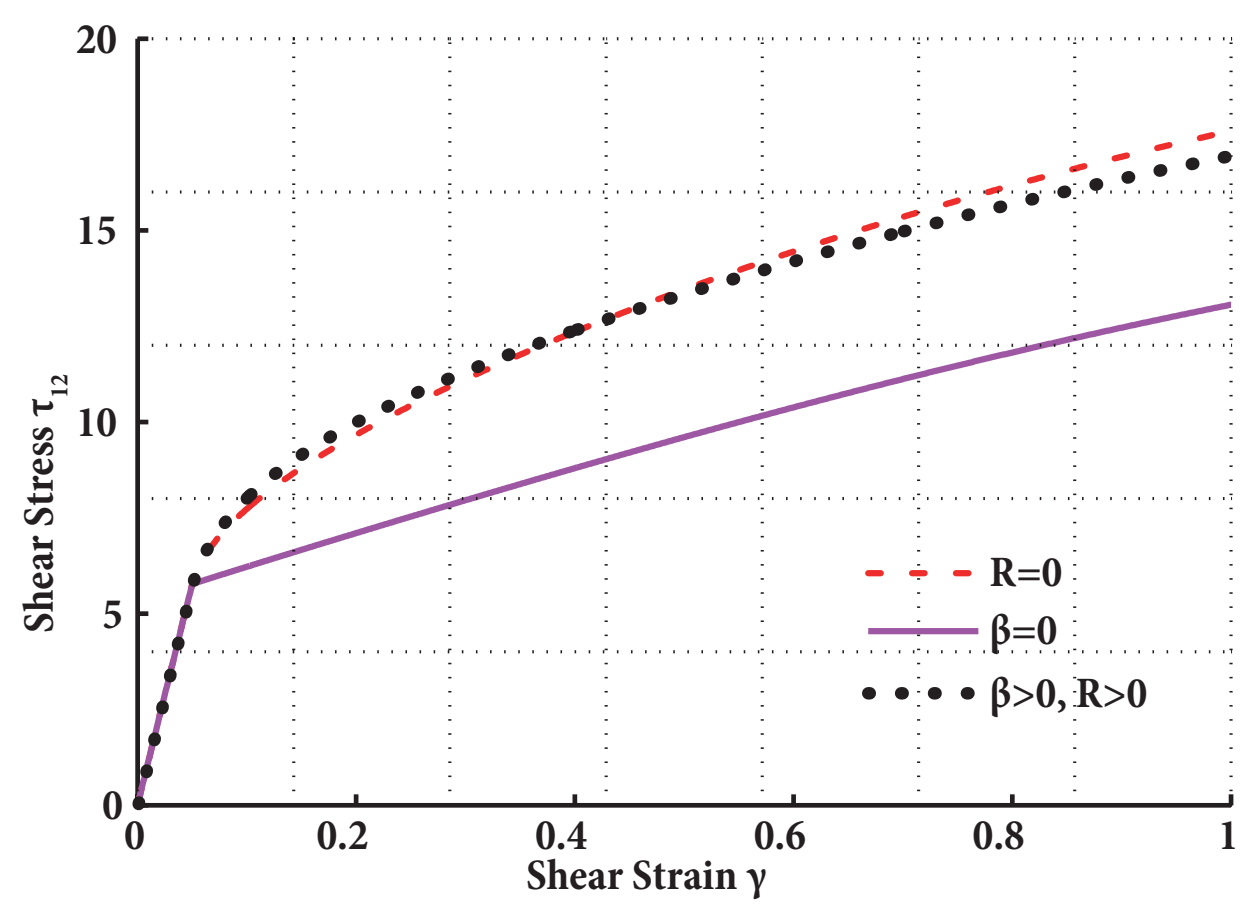

Fig. 3. Simple shear for the limiting cases Shear stress vs. shear strain strain 


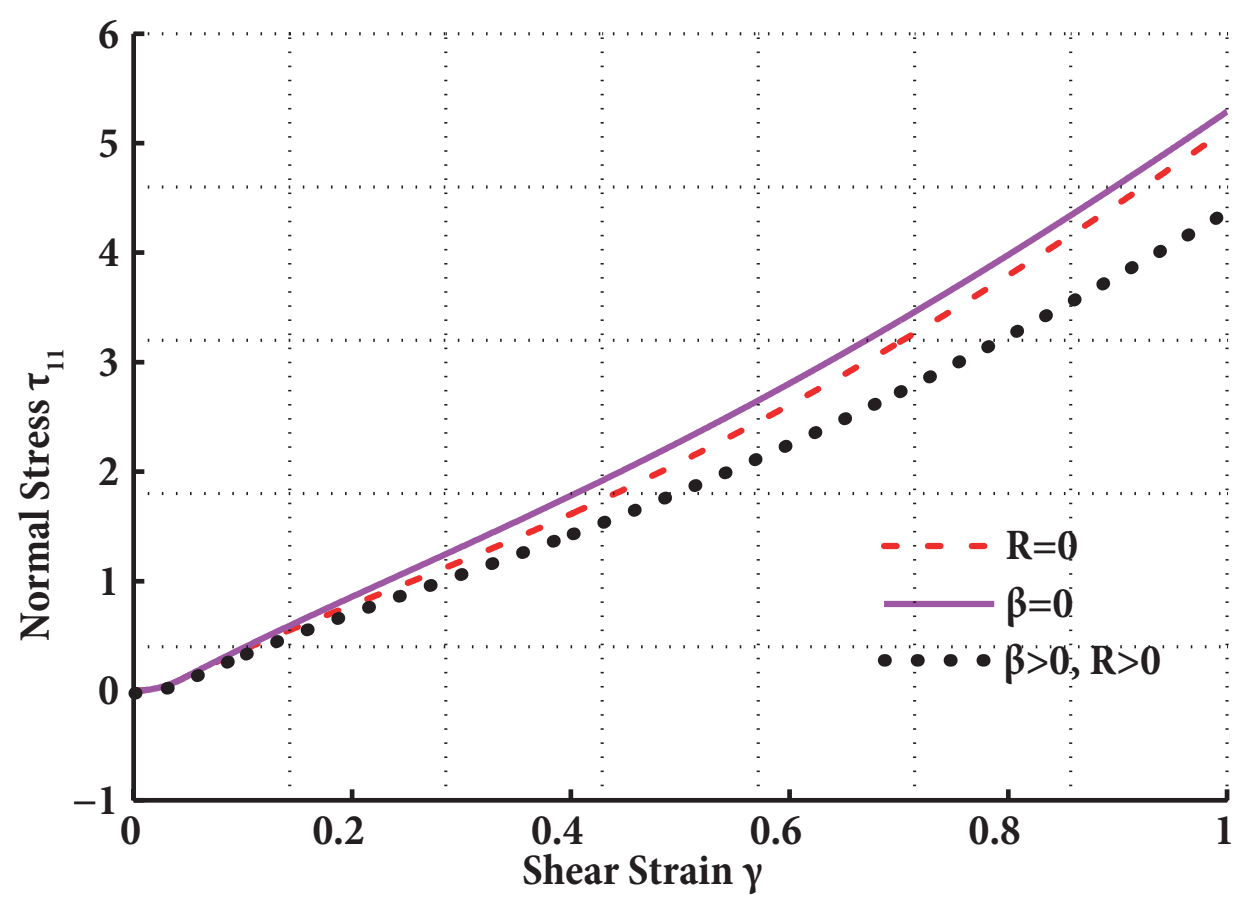

Fig. 4. Simple shear for the limiting cases Normal stress vs. shear strain strain 


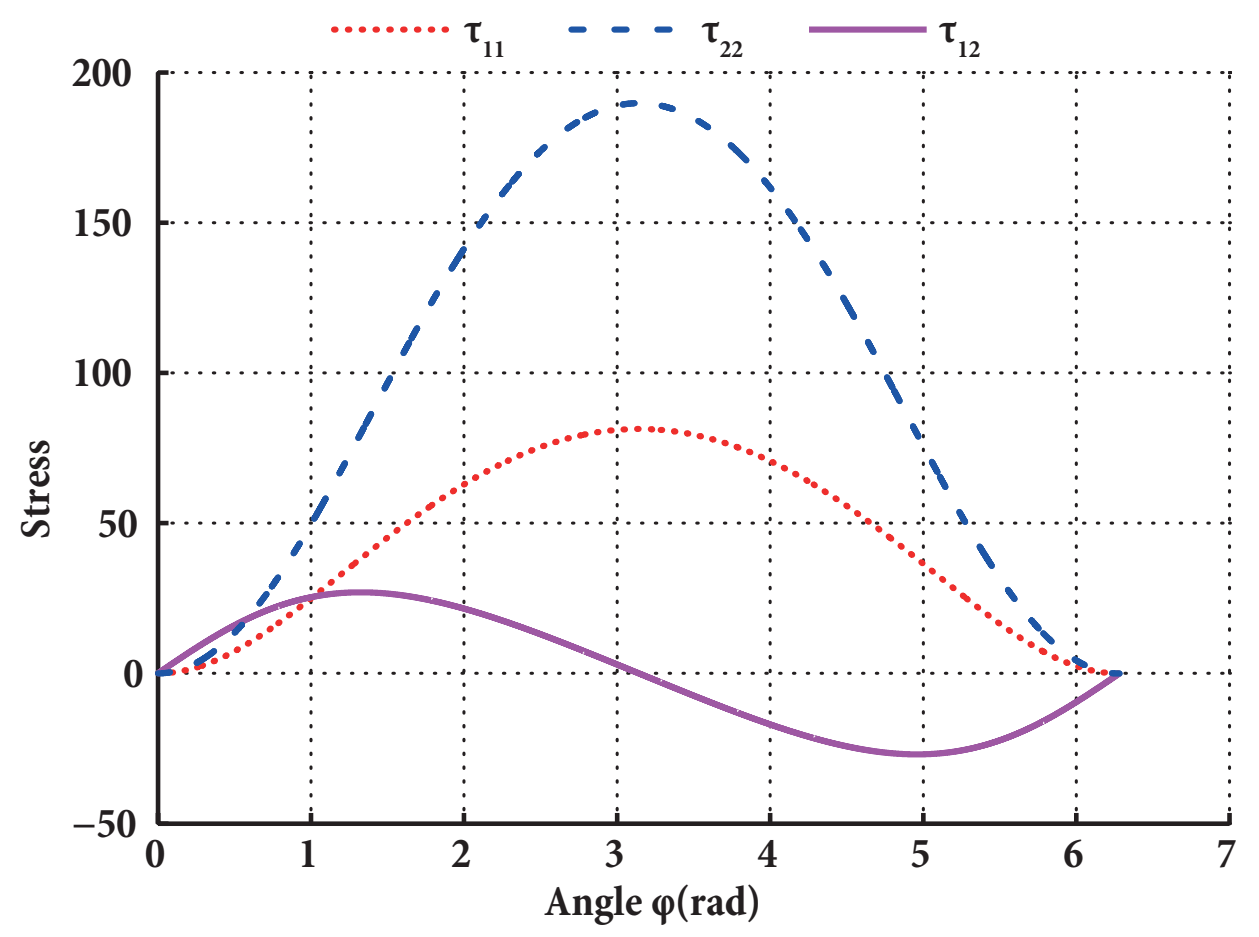

Fig. 5. Response under a strain cycle (elastic case) Stresses vs. angle of rotation 


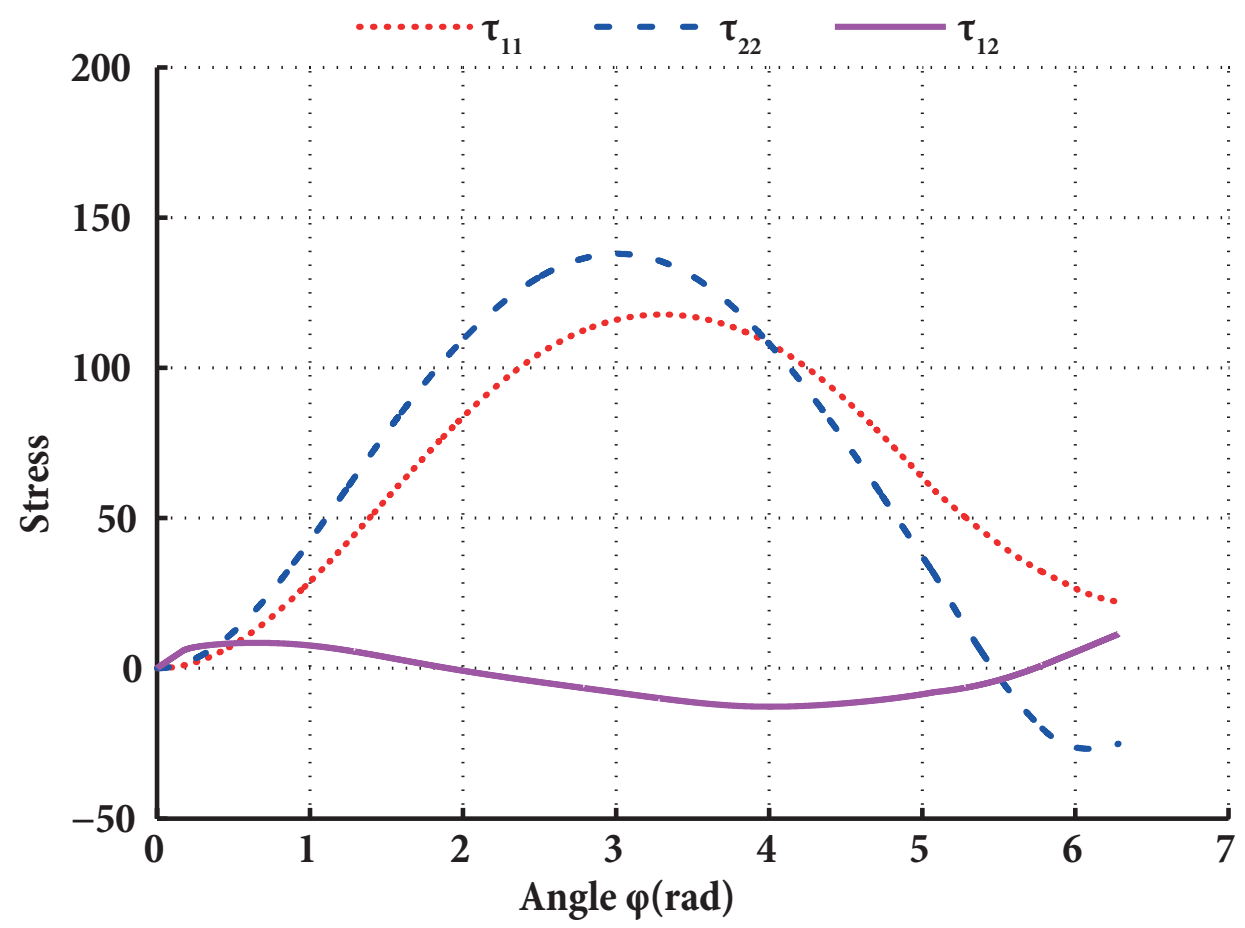

Fig. 6. Response under a strain cycle (elastic-plastic case). Stresses vs. angle of rotation 


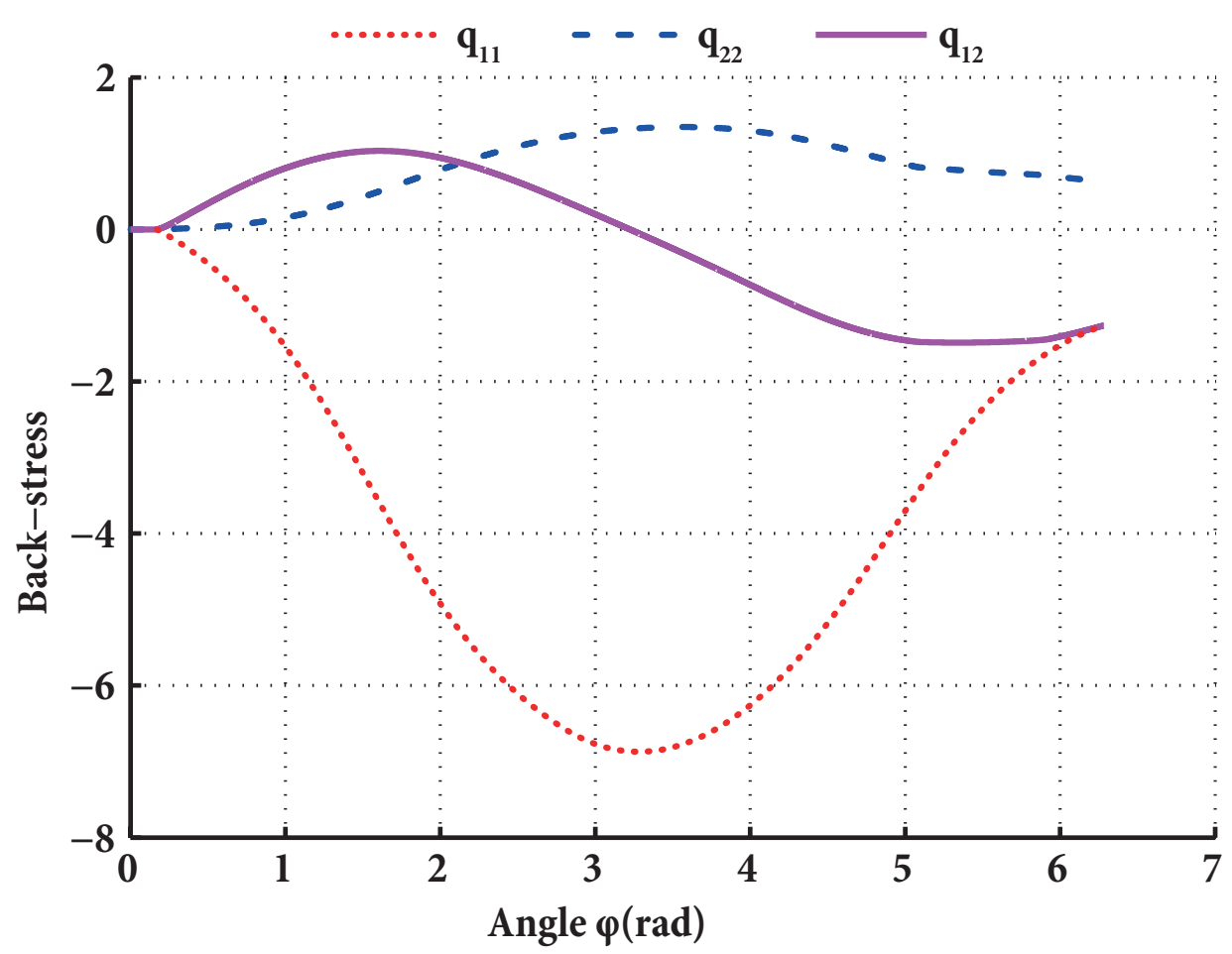

Fig. 7. Response under a strain cycle (elastic-plastic case). Back stresses vs. angle of rotation 


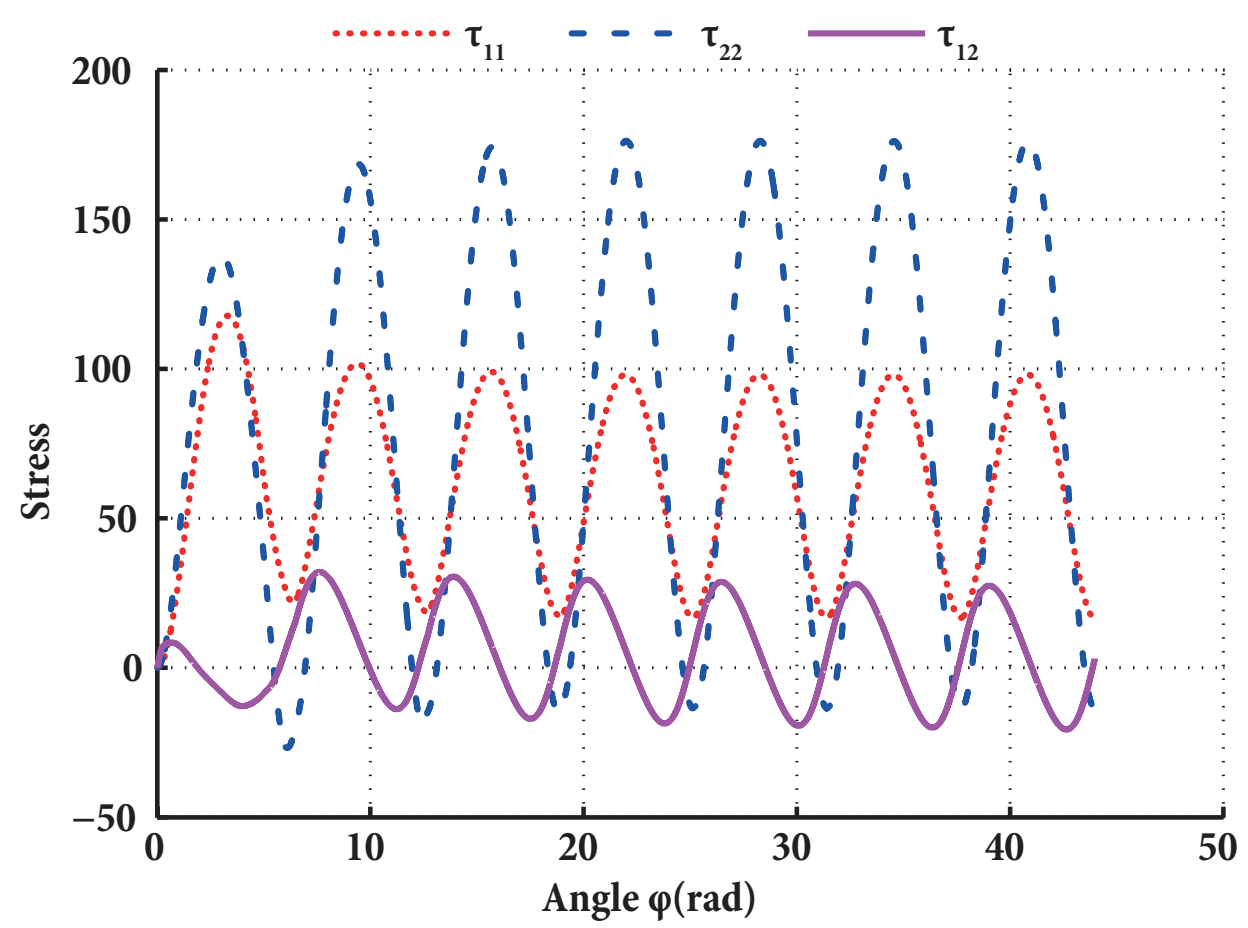

Fig. 8. Response under a strain cycle (elastic-plastic case; $R=30.00$ ) Stresses vs. angle of rotation 


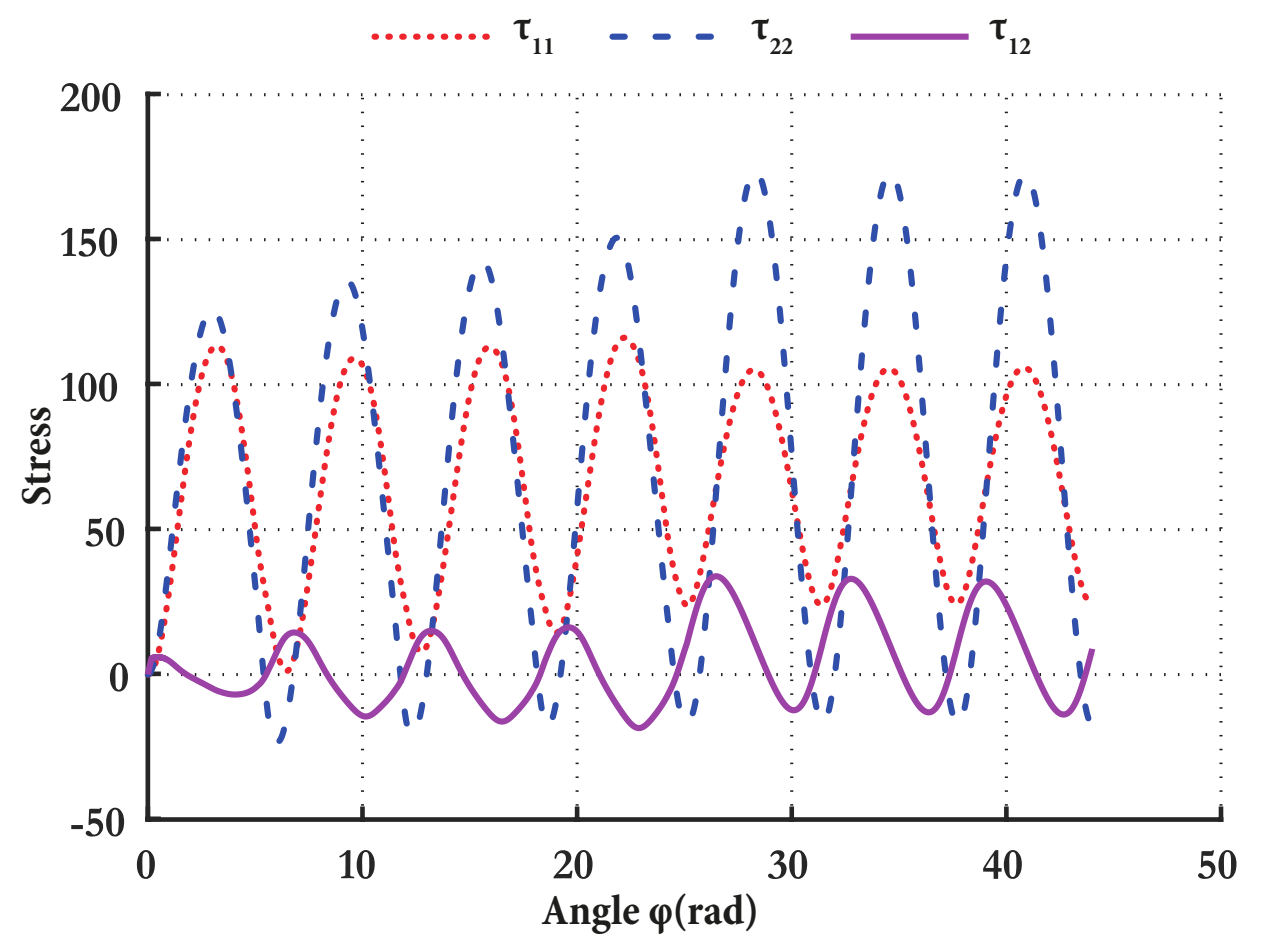

Fig. 9. Response under a strain cycle (elastic-plastic case; $R=30.00, \beta=0$ ) Stress vs Angle of rotation 